\title{
氮添加对山西太岳山天然油松林主要植物叶片性 状的影响
}

\section{肖 迪 ${ }^{1}$ 王晓洁 ${ }^{1}$ 张 凯 ${ }^{1}$ 何念鹏 ${ }^{2}$ 侯继华 ${ }^{1 *}$}

${ }^{1}$ 北京林业大学资源与生态系统过程北京市重点实验室, 北京 100083 ; ${ }^{2}$ 中国科学院地理科学与资源研究所生态系统网络观测与模拟重点实验室, 北京 100101

摘 要 为探讨植物性状对大气氮沉降的响应与适应机制, 该文以中国特有的、在北方温性针叶林中广泛分布的天然油松 (Pinus tabuliformis)林为研究对象, 在2009-2013年开展了氮添加对植物叶片性状影响的野外控制试验, 4 个氮添加浓度分别为 $0 \mathrm{~kg} \cdot \mathrm{hm}^{-2} \cdot \mathrm{a}^{-1}$ (CK)、50 kg$\cdot \mathrm{hm}^{-2} \cdot \mathrm{a}^{-1}$ (低氮)、 $100 \mathrm{~kg} \cdot \mathrm{hm}^{-2} \cdot \mathrm{a}^{-1}$ (中氮)和 $150 \mathrm{~kg} \cdot \mathrm{hm}^{-2} \cdot \mathrm{a}^{-1}$ (高氮)。试验过程中分别测定了油松、蒙 古栋(Quercus mongolica)、茶条槭(Acer ginnala)、毛榛(Corylus mandshurica)、沙梾(Cornus bretschneideri)、绣线菊(Spiraea salicifolia)、金银忍冬(Lonicera maackii)、羊须草(Carex callitrichos)、龙常草(Diarrhena mandshurica)、大火草(Anemone tomentosa)和玉竹(Polygonatum odoratum)等11种主要植物的 9 种叶片性状, 包括叶厚度 $(L T)$ 、比叶面积(SLA)、干物质含量 $(L D M C)$ 、叶氮含量 $(L N C)$ 、叶磷含量 $(L P C)$ 等。结果表明: 1)在氮添加影响下, 玉竹等个别物种的 $L T$ 和 $S L A$ 、绣线菊等部分物 种的叶面积 $(L A)$ 和 $L D M C$ 差异显著, 上述所有物种的 $L N C$ 与大多数物种的叶绿素含量 $(C C) 、 L P C$ 显著增加, 油松等 9 种植物叶 片 $\mathrm{N}: \mathrm{P}$ 发生显著变化，不同年龄、不同类型的植物叶片对氮添加的响应不同。2)叶性状之间普遍存在显著相关性，如SLA与 与NC 和 $L P C$ 极显著正相关, $L T$ 与 $L N C$ 和 $L P C$ 极显著负相关, 且相关性随氮添加强度变化。3) 11种植物的叶片特征空间分布规律与叶 经济谱的描述一致，氮添加使植物在特征空间中的位置向叶片薄、生长快、叶寿命短的“快速投资-收益型”一端发生移动; 在 垂直方向上, 阔叶乔木、灌木及草本的位置与针叶乔木的移动方向相反。当环境改变时, 植物会改变生存策略, 调整资源分 配, 从而保证物种间相对位置和群落整体结构的稳定性。叶经济谱的形成不依赖于环境的变化, 而是植物一种固有的属性。 关键词 大气氮沉降; 叶经济谱; 叶片功能性状; 天然油松林; 氮添加

引用格式: 肖迪, 王晓洁, 张凯, 何念鹏, 侯继华 (2016). 氮添加对山西太岳山天然油松林主要植物叶片性状的影响. 植物生态学报, 40, 686-701. doi: 10.17521/cjpe.2015.1043

\section{Effects of nitrogen addition on leaf traits of common species in natural Pinus tabuliformis fo- rests in Taiyue Mountain, Shanxi Province, China}

XIAO Di ${ }^{1}$, WANG Xiao-Jie ${ }^{1}$, ZHANG Kai ${ }^{1}$, HE Nian-Peng ${ }^{2}$, and HOU Ji-Hua ${ }^{1 *}$

${ }^{1}$ Key Laboratory for Forest Resources \& Ecosystem Processes of Beijing Forestry University, Beijing 100083, China; and ${ }^{2}$ Key Laboratory of Ecosystem Network Observation and Modeling, Institute of Geographic Sciences and Natural Resources Research, Chinese Academy of Sciences, Beijing 100101, China

\section{Abstract}

Aims Our objectives were to explore the effects of simulated nitrogen deposition on leaf traits and ecological stoichiometry characteristics of common species in natural Pinus tabuliformis forests.

Methods We conducted the experiment of nitrogen (N) addition from 2009 to 2013 in the natural Pinus tabuliformis forests in Taiyue Mountain, Shanxi, China. The levels of N addition were 0 (control), 50 (low-N), 100 (medium-N) and 150 (high-N) $\mathrm{kg} \cdot \mathrm{hm}^{-2} \cdot \mathrm{a}^{-1}$, respectively. Eleven common plant species in $1220 \mathrm{~m} \times 20 \mathrm{~m}$ plots were selected, including Pinus tabuliformis, Quercus mongolica, Acer ginnala, Corylus mandshurica, Cornus bretschneideri, Spiraea salicifolia, Lonicera maackii, Carex callitrichos, Diarrhena mandshurica, Anemone tomentosa, and Polygonatum odoratum. Nine leaf traits were measured, including leaf thickness (LT), specific leaf area $(S L A)$, leaf dry matter content $(L D M C)$, leaf nitrogen content $(L N C)$, leaf phosphorus content (LPC), and other four.

Important findings We found that: 1) LT and SLA of Polygonatum odoratum significantly differed among four levels of $\mathrm{N}$ addition. Leaf area ( $L A)$ and $L D M C$ of several species, such as Spiraea salicifolia, had significant

收稿日期Received: 2015-01-29 接受日期Accepted: 2015-12-27

* 通信作者Author for correspondence (E-mail: houjihua@bjfu.edu.cn) 
difference among the $\mathrm{N}$ addition concentration. $L N C$ of all species, chlorophyll content $(C C)$ and $L P C$ of most species increased significantly with the addition of $\mathrm{N}$. Leaf N:P of 9 species varied significantly, and leaves with different types and ages showed different responses to $\mathrm{N}$ addition. 2) Leaf traits were significantly correlated with each other. For instance, SLA was significantly positively correlated with $L N C$ and $L P C$. In contrast $L T$ was negatively connected with $L N C$ and $L P C$. In addition, the degree of correlation changed with the level of $\mathrm{N}$ addition. 3) The pattern of species distribution in leaf trait space was consistent with the prediction from the theory of Leaf Economic Spectrum (LES). $\mathrm{N}$ addition drove species moving along axis 1 in the trait space, and propelled them towards different directions along axis 2, which indicated that these species tended to take the "fast investment-return” strategy. These results suggested that with the change of environmental conditions, plants changed their survival strategy and adjusted resource allocation to maintain the stability of communities. This is the inherent characteristic of plants, thus the formation of LES did not depend on the environment change.

Key words atmospheric nitrogen deposition; leaf economic spectrum; leaf functional traits; natural Pinus tabuliformis forest; nitrogen addition

Citation: Xiao D, Wang XJ, Zhang K, He NP, Hou JH (2016). Effects of nitrogen addition on leaf traits of common species in natural Pinus tabuliformis forests in Taiyue Mountain, Shanxi Province, China. Chinese Journal of Plant Ecology, 40, 686-701. doi: 10.17521/cjpe.2015.1043

自工业革命以来, 大气氮沉降呈现急剧增加的 趋势(Holland et al., 1999; Zheng et al., 2002; Adams et al., 2004; Suding et al., 2005), 这直接影响到人类 和生态系统的健康、温室气体的平衡及生物的多样 性, 引起了科学家们的广泛关注与担忧(Vitousek et al., 1997; Richter et al., 2005; Clark \& Tilman, 2008; Liu et al., 2013)。研究表明, 氮素作为陆地生态系统 中最主要的限制元素(贺金生和韩兴国, 2010), 对森 林植物有重要影响(李德军等, 2005)。在其他养分成 为限制因子之前, 氮的增加可以提高植物的净初级 生产力(Aber et al., 1989; Tamm, 1990), 但长期、连 续、过量的氮输入会降低植物的净光合速率和净初 级生产力, 引起森林生产力的下降和森林生态系统 功能的退化(Ulrich, 1995; Fenn et al., 1996; Magill et al., 2004; 方运霆等, 2005), 这被认为是森林衰退的 主要原因(Nihlgard, 1985; Bobbink et al., 1998; 张维 娜和廖周瑜, 2009)。因此, 研究大气氮沉降对森林 植物的影响具有十分重要的意义。

植物通常会对自身资源进行配置、补偿和平衡, 以最大限度地减小环境变化对植物的不利影响, 植 物功能性状(functional traits)就是这种调节机制的具 体体现(Comelissen et al., 2003), 因此成为探索植物 与环境之间关系的重要纽带, 也为预测在全球变化 背景下的生态系统响应提供了方法和依据(Wright et al., 2005)。其中, 叶片性状对气候变化十分敏感, 能较为准确地反映植物对气候变化的响应与适应机 制(Royer et al., 2010; Scoffoni et al., 2011)。研究表
明, 氮沉降可导致植物比叶面积 $(S L A)$ 、叶片氮含量 $(L N C)$ 、叶片磷含量 $(L P C)$ 及氮磷比 $(\mathrm{N}: \mathrm{P})$ 等发生显著 改变(Pontes et al., 2007; 黄菊荣等, 2009; Ordoñez et al., 2009; Rose et al., 2013; 王常顺和汪诗平, 2015)。

Wright等(2004)以叶片的形态、生理、化学性状 对叶片的资源权衡策略进行量化, 发现植物叶片性 状沿着一条连续变化的功能性状组合谱(即叶经济 谱, LES)有序排列, 其一端代表着比叶重小、含氮量 高、光合速率大、呼吸速率高、叶寿命短的“快速投 资-收益型”策略; 而另一端代表着寿命长、比叶重 大、含氮量、光合速率和呼吸速率都偏低的“缓慢投 资-收益型”策略。叶经济谱为解释碳、氮元素在植 物体内的固定和利用、营养物质的流动, 以及植被 的分布如何随环境条件变化而改变等问题提供了理 论依据(Royer, 2009; 陈芗婷和许振柱, 2014)。之后, 关于植物的资源权衡关系及全球尺度上植物叶片性 状随环境的变化规律的研究大量涌现(Reich et al., 2003; Freschet et al., 2010; Laughlin, 2011; Osnas et al., 2013)。

然而, 关于植物叶经济谱的形成动力仍存在较 大的争议：一方面, Maire等 (2013) 和 Blonder等 (2013) 认为环境梯度引发植物资源权衡发挥作用, 通过自我调节响应环境变化, 因此, 叶经济谱受环 境因子主导; 另一方面, Wright等(2004)、Leishman 等(2007)、Wright和Sutton-Grier (2012)等则认为植物 的生活史策略和叶经济谱是独立演变的, 受气候变 化和群落类型的影响微之甚微。基于全球尺度的“叶 
经济谱”假说有一个重要前提假设, 是物种性状的 单一平均值具有足够的代表性。然而, 这一重要假 设的有效性并没有得到充分验证。众所周知, 达尔 文生物进化论最基本的一点就是同种个体在许多性 状上的差异是生物适应环境而进化的基础。利用单 一性状值来描述不同地区的同一个物种掩盖了该物 种沿环境梯度某一或多个性状的巨大变异(Albert et al., 2010a)。这种忽视种内性状差异的观点将严重限 制我们对生物与环境关系的理解, 极大地阻碍现有 模型的预测能力(McGill et al., 2006; Wright \& Sutton-Grier, 2012)。因此, 在控制条件下研究局域种库 内物种的性状如何响应环境变化可以尽量避免其他 生态因子的干扰, 能更有效地揭示植物对环境变化 的适应机制, 而目前这类研究还较为少见(Albert et al., 2010b, 2011)。

油松(Pinus tabuliformis)是我国的特有种, 在北 方地区分布广泛。山西太岳山地区素有“油松之乡” 的美称, 有关该地区大气氮沉降对森林植物功能性 状影响的研究鲜有报道。本研究试图以该地天然油 松林为研究对象, 通过人工氮添加控制试验对自然 条件下的氮沉降进行模拟, 观察、预测氮素变化对 局域种库内植物叶片功能性状的影响, 并为解释植 物应对环境变化的响应机制提供理论依据。本研究 的主要目的是: (1)检验氮添加是否对局域种库内植 物的叶片性状产生显著影响, 并分析性状变异的来 源; (2)比较不同种类植物叶片对氮沉降响应的差异; (3)检验叶片性状间的相关性、叶经济谱存在与否及 其形成动力; (4)探究天然油松林内主要植物叶片性 状在经济谱中的位置是否受到氮添加的影响。

\section{1 材料和方法}

\section{1 研究区概况}

本研究区位于山西省长治市沈源县, 太岳山森 林经营局灵空山自然保护区内 $\left(36.00^{\circ}-37.08^{\circ} \mathrm{N}\right.$, $111.75^{\circ}-112.55^{\circ} \mathrm{E}$, 海拔1 150-2 $\left.088 \mathrm{~m}\right)$, 是我国暖 温带落叶淍叶林典型代表地区。该研究区属暖温带 半干旱大陆性季风气候, 年平均气温 $6.2{ }^{\circ} \mathrm{C}$, 四季 分明, 春季多风。年降水量662 mm, 降水集中在7-9 月, 占全年降水量的 $60 \%$ 以上。相对湿度 $60 \%-65 \%$, 年日照时间 $2600 \mathrm{~h}$, 年无霜期110-125天。土壤以褐 土和棕壤为主。油松、蒙古栎(Quercus mongolica)、 山杨(Populus davidiana)、白桦(Betula platyphylla)
是本研究区最具代表性的树种, 灌木主要有毛榛 (Corylus mandshurica)、沙 棘 (Hippophae rhamnoides)、黄刺玫(Rosa xanthina)、胡枝子(Lespedeza bicolor)等, 草类有薹草(Carex spp.)、莎草(Cyperus spp.)、蒿类等。

\section{2 试验设计}

以欧洲氮饱和实验 NITREX 项目 (Wright \& Rasmussen, 1998)和北美哈佛森林(Harvard Forest) 等(Aber et al., 1998)研究设计为参照, 在未被破坏 的天然油松林 $\left(112.02^{\circ}-112.25^{\circ} \mathrm{E}, 36.52^{\circ}-36.72^{\circ} \mathrm{N}\right.$, 平均海拔 $1500 \mathrm{~m}$ )中建立 12 个 $20 \mathrm{~m} \times 20 \mathrm{~m}$ 的固定试 验样地, 设置对照 $\left(C K, 0 \mathrm{~kg} \cdot \mathrm{hm}^{-2} \cdot \mathrm{a}^{-1}\right)$ 、低氮( $(\mathrm{LN}, 50$ $\left.\mathrm{kg} \cdot \mathrm{hm}^{-2} \cdot \mathrm{a}^{-1}\right)$ 、中氮 $\left(\mathrm{MN}, 100 \mathrm{~kg} \cdot \mathrm{hm}^{-2} \cdot \mathrm{a}^{-1}\right)$ 和高氮( $\mathrm{HN}$, $150 \mathrm{~kg} \cdot \mathrm{hm}^{-2} \cdot \mathrm{a}^{-1}$ ) 4 个处理, 3 次重复。样地林分特征 及0-20 cm土壤理化性质详见表1。从2009年5月起, 用尿素 $\left(\mathrm{CO}\left(\mathrm{NH}_{2}\right)_{2}\right)$ 作为氮源, 将年施用量平均分成 12 等份, 每月下旬分别将所需的尿素溶解于 $2 \mathrm{~L}$ 水 中, 用背式喷雾器在对应处理的 $20 \mathrm{~m} \times 20 \mathrm{~m}$ 样地内 均匀喷酒(对照组喷施等量的水), 其他水源来自自 然降水。

\section{3 研究对象}

根据群落调查结果计算样地内各植物种的重要 值, 选取出现频度最高、重要值大于 0.05 的 11 个物种 进行研究, 包括3种乔木、4种灌木和 4 种草本, 分别 是针叶树种油松 $(\mathrm{Pt})$ 、阔叶树种蒙古栋 $(\mathrm{Qm})$ 和茶条 槭 (Acer ginnala, Ag); 毛榛 (Cm)、沙梾 (Cornus bretchneideri, $\mathrm{Cb}$ )、绣线菊 (Spiraea salicifolia) 和金银 忍冬(Lonicera maackii, Lm); 羊须草(Carex callitrichos, Cc)、龙常草(Diarrhena mandshurica, Dm)、大 火草(Anemone tomentosa, At)和玉竹(Polygonatum odoratum, Po)。

\section{4 样品采集}

样品采集在2013年7月完成。在每个样地中, 每 个物种随机选择5株(部分草本一从视为一株)长势 良好的成熟个体。每株随机采集20片完全展开、没 有病虫害的外层成熟叶片(油松当年生叶与多年生 叶分别采样), 置于两片湿润滤纸之间放入自封袋, 用装有冰袋的保温箱尽快带回实验室。

\section{5 性状测定}

采集的样品当天进行处理。先将所有植物叶片 表面洗净擦干, 剪去叶柄。采用SPAD 502-PLUS手 持式叶绿素仪(Konica Minolta, Tokyo, Japan)测定各 
表1 天然油松林各处理样地的林分特征和土壤理化性质

Table 1 Stand characteristics and soil physical-chemical properties of the nitrogen-loaded plots in the natural forest of Pinus tabuliformis

\begin{tabular}{|c|c|c|c|c|c|c|c|c|c|c|}
\hline \multirow[t]{2}{*}{$\begin{array}{l}\text { 处理 } \\
\text { Treatment }\end{array}$} & \multicolumn{5}{|c|}{$\begin{array}{c}\text { 林分特征 } \\
\text { Stand characteristics }\end{array}$} & \multicolumn{5}{|c|}{$\begin{array}{c}\text { 土壤理化性质 } \\
\text { Physical-chemical properties of soil }\end{array}$} \\
\hline & $\begin{array}{l}\text { 林龄 } \\
\text { Stand } \\
\text { age } \\
\text { (a) }\end{array}$ & $\begin{array}{c}\text { 密度 } \\
\text { Density } \\
\left(\text { plant } \cdot h m^{-2} \text { ) }\right.\end{array}$ & $\begin{array}{c}\text { 平均胸径 } \\
\text { Mean diameter } \\
\text { at breast height } \\
(\mathrm{cm})\end{array}$ & $\begin{array}{c}\text { 平均株高 } \\
\text { Mean plant } \\
\text { height } \\
\text { (m) }\end{array}$ & $\begin{array}{l}\text { 坡度 } \\
\text { Slope } \\
\left({ }^{\circ}\right)\end{array}$ & $\begin{array}{c}\text { 土壤容重 } \\
\text { Soil volume } \\
\text { weight } \\
\left(\mathrm{g} \cdot \mathrm{cm}^{-3}\right) \\
\end{array}$ & $\begin{array}{l}\text { 土壤pH } \\
\text { Soil pH }\end{array}$ & $\begin{array}{c}\text { 土壤有机碳含量 } \\
\text { Soil organic car- } \\
\text { bon content } \\
\left(\mathrm{mg} \cdot \mathrm{g}^{-1}\right)\end{array}$ & $\begin{array}{c}\text { 土壤氮含量 } \\
\text { Soil nitrogen } \\
\text { content } \\
\left(\mathrm{mg} \cdot \mathrm{g}^{-1}\right) \\
\end{array}$ & $\begin{array}{c}\text { 土壤磷含量 } \\
\text { Soil phosphorus } \\
\text { content } \\
\left(\mathrm{mg} \cdot \mathrm{g}^{-1}\right)\end{array}$ \\
\hline 对照 Control & 75 & 1267 & 23.9 & 17.7 & 24 & 0.94 & 7.12 & 45.30 & 1.70 & 0.43 \\
\hline 低氮 Low-N & 75 & 1567 & 20.6 & 17.8 & 21 & 0.97 & 7.12 & 61.02 & 2.14 & 0.38 \\
\hline 中氮 Medium-N & 75 & 1208 & 23.5 & 17.4 & 25 & 1.07 & 7.19 & 42.14 & 1.91 & 0.38 \\
\hline 高氮 High-N & 75 & 1225 & 23.4 & 19 & 23 & 1.06 & 7.28 & 36.08 & 2.25 & 0.44 \\
\hline
\end{tabular}

Control, 氮添加浓度为 $0 \mathrm{~kg} \cdot \mathrm{hm}^{-2} \cdot \mathrm{a}^{-1} ; \mathrm{Low}-\mathrm{N}$, 氮添加浓度为 $50 \mathrm{~kg} \cdot \mathrm{hm}^{-2} \cdot \mathrm{a}^{-1} ;$ Medium-N, 氮添加浓度为 $100 \mathrm{~kg} \cdot \mathrm{hm} \mathrm{m}^{-2} \cdot \mathrm{a}^{-1} ; \mathrm{High}-\mathrm{N}$, 氮添加浓度为 150 $\mathrm{kg} \cdot \mathrm{hm}^{-2} \cdot \mathrm{a}^{-1}$ 。本表部分数据引自李化山等(2014)。

Control, $0 \mathrm{~kg} \cdot \mathrm{hm}^{-2} \cdot \mathrm{a}^{-1}$ nitrogen; Low-N, $50 \mathrm{~kg} \cdot \mathrm{hm}^{-2} \cdot \mathrm{a}^{-1}$ nitrogen; Medium-N, $100 \mathrm{~kg} \cdot \mathrm{hm}^{-2} \cdot \mathrm{a}^{-1}$ nitrogen; High-N, $150 \mathrm{~kg} \cdot \mathrm{hm}{ }^{-2} \cdot \mathrm{a}^{-1}$ nitrogen. The data was partly derived from Li et al. (2014).

叶片的叶绿素含量 (CC, 单位SPAD, 每片测量3个 不同位置), 取平均值。每种叶片 5 个为一组沿主脉 方向整齐叠放, 沿主脉约 $0.25 \mathrm{~cm}$ 处均匀选择 3 个点, 用精度 $0.01 \mathrm{~mm}$ 的数显游标卡尺测量厚度值, 取其 平均值即为叶厚度 $(L T, \mu \mathrm{m})$ 。阔叶树种、灌木、草本 的叶片用HP Scanjet G3110扫描仪(Hewlett-Packard Development Company, Palo Alto, USA)扫描后, 应 用 Image $\mathrm{J}$ 图像处理软件 (National Institutes of Health, Bethesda, USA)计算每种植物叶片的平均叶 面积 $\left(L A, \mathrm{~cm}^{2}\right)$; 而针叶树种油松叶面积的测量, 首 先用数显游标卡尺和直尺测量单松针的长度 $L$ $(\mathrm{cm}) 、$ 宽度 $W(\mathrm{~mm}) 、$ 厚度 $D(\mathrm{~mm})$, 取平均值, 再根 据公式 $L A\left(\mathrm{~cm}^{2}\right)=0.01 \times[\pi(W / 2+D)+2 W] \times L$ 计算 得到单针叶面积(李轩然等, 2007)。用电子天平(精度 为 $0.01 \mathrm{~g}$ )称取每物种 20 片叶片的饱和鲜质量 (SM, $\mathrm{g}$ ), 然后在 $85{ }^{\circ} \mathrm{C}$ 下烘干 $48 \mathrm{~h}$ 至恒质量, 分别称量干 质量(DM, g)。再据此计算其他指标: 比叶面积(SLA, $\left.\mathrm{m}^{2} \cdot \mathrm{kg}^{-1}\right)=L A / D M$; 叶干物质含量 $\left(L D M C, \mathrm{~g} \cdot \mathrm{kg}^{-1}\right)=$ $D M / S M$ 。将烘干叶片磨粉过细篎后, 采用重铬酸钾 外加热法测有机碳含量 $\left(L C C, \mathrm{mg} \cdot \mathrm{g}^{-1}\right)$; 经 $\mathrm{H}_{2} \mathrm{SO}_{4}{ }^{-}$ $\mathrm{H}_{2} \mathrm{O}_{2}$ 法消煮后, 用凯氏定氮法测定全氮含量 $(L N C$, $\left.\mathrm{mg} \cdot \mathrm{g}^{-1}\right)$, 钿锑抗比色法测定全磷含量 (LPC, $\left.\mathrm{mg} \cdot \mathrm{g}^{-1}\right)$ 。

\section{6 数据分析}

叶性状数值由算术平均值和标准误差来表示。 采用单因素方差分析检验氮添加对植物功能性状的 影响, 采用Fisher最小显著差数(LSD)法检验各性状 在不同处理间的显著性差异。为满足正态分布及方 差齐性的假设, 对所有数据进行 $\log _{10}$ 对数转换后, 利用Pearson相关性探讨叶性状间的相关关系。运用
主成分分析(PCA)对不同植物多个叶性状进行综合 分析, 并据此分析植物在叶经济谱上的分布状况以 及氮添加对植物性状变异的解释力。数据分析与图 表制作采用SPSS 18.0 (SPSS, Chicago, USA)和 Sigma Plot 12.0 (Systat Software, San Jose, USA)软 件完成。

\section{2 实验结果}

\section{1 氮添加对叶片形态、叶绿素含量和叶干物质含 量的影响}

结果表明, 氮添加对不同物种的叶片形态、叶 绿素含量和叶干物质含量有不同程度的影响(图1)。 随着氮添加强度的增加, 龙常草和大火草的叶厚度 $(L T)$ 显著减小, 玉竹的 $L T$ 则显著增大(图1A); 氮添 加对蒙古柇、毛榛、绣线菊、大火草和玉竹的叶面 积 $(L A)$ 有显著影响, 随氮添加它们的叶面积 $(L A)$ 呈 增大趋势(图1B), 但蒙古楿和玉竹的 $L A$ 在高氮水平 回降; 茶条枫比叶面积(SLA)先增后减, 玉竹SLA显 著减小(图1C); 蒙古栋、茶条枫等8种植物的叶绿素 含量 $(C C)$ 随氮添加强度增大而显著升高, 其中茶条 枫、金银忍冬和玉竹在高氮水平回降(图1D); 油松 当年生叶和玉竹的叶干物质含量 $(L D M C)$ 显著升高, 绣线菊和大火草的 $L D M C$ 在中氮水平显著低于其他 水平(图1E)。将油松当年生叶片(Pt-cy)与往年生叶 片(Pt-fy)进行横向比较得出, 当年生叶具有较小的 $L T 、 L A 、 C C 、 L D M C$ 和较大的 $S L A$, 且 $L D M C$ 受氮 添加的影响更为显著(图1)。

\section{2 氮添加对叶片碳、氮、磷元素含量的影响}

分析表明, 氮添加对植物叶碳含量 $(L C C)$ 没有 显著影响(图2A)。而11种植物的叶氮含量 $(L N C)$ 均随 
690 植物生态学报 Chinese Journal of Plant Ecology 2016, 40 (7): 686-701
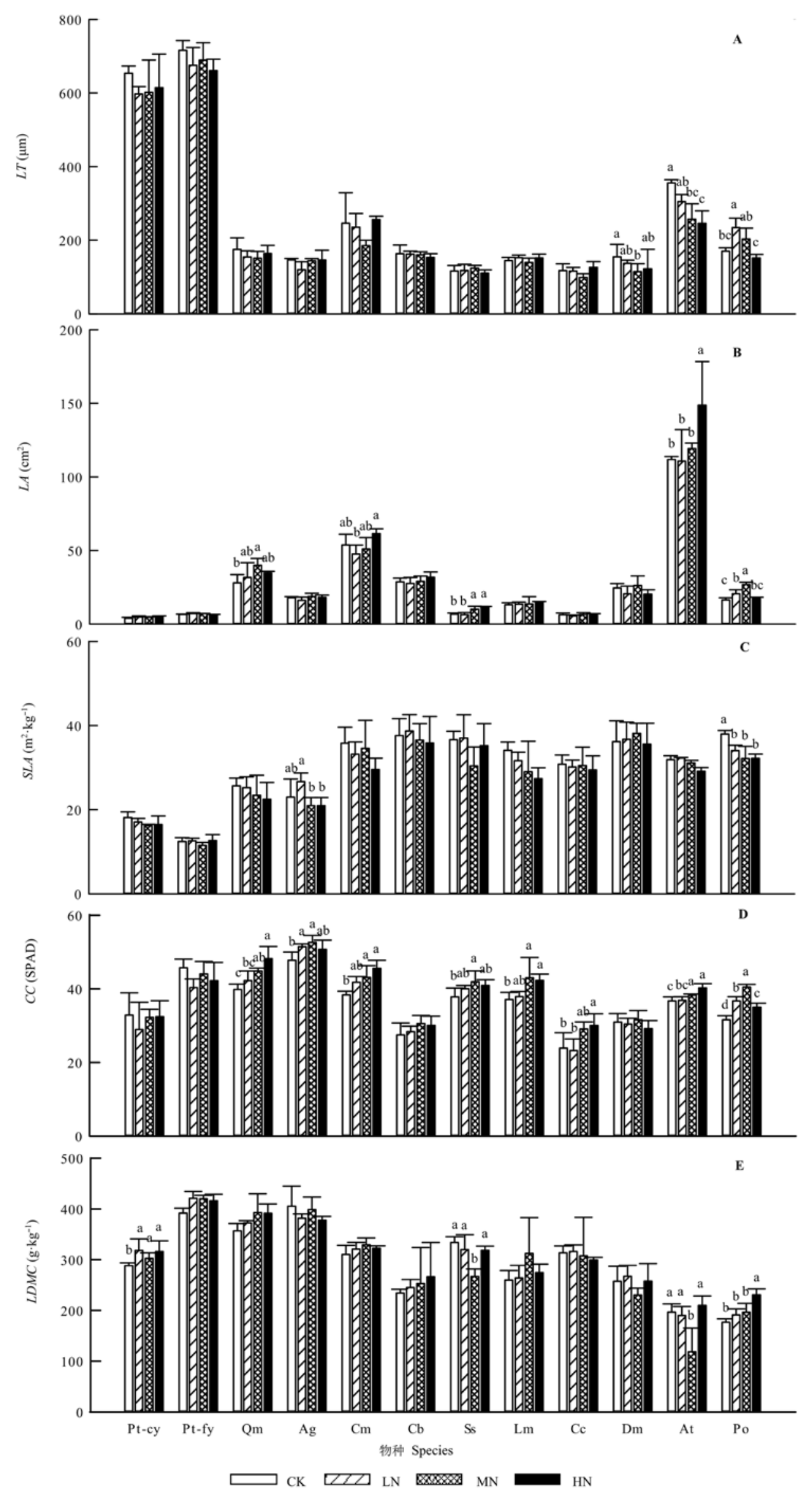
着氮添加强度增大而显著升高(图2B)。叶磷含量 $(L P C)$ 对氮添加的响应十分显著, 油松当年生叶 $L P C$ 在中氮水平显著升高, 在高氮水平又显著降低 至对照水平以下; 油松往年生叶无显著变化; 其他 植物的 $L P C$ 均随氮添加强度增加而显著升高, 部分 在高氮水平受到抑制(图2C)。蒙古栎和羊须草 $\mathrm{N}: \mathrm{P}$ 对氮添加响应不显著, 油松、茶条枫和毛榛 $\mathrm{N}: \mathrm{P}$ 受氮 添加影响先增后减(氮添加组高于对照组), 大火草 $\mathrm{N}: \mathrm{P}$ 显著增大, 其他植物叶片 $\mathrm{N}: \mathrm{P}$ 均显著减小 (图 2D)。油松当年生叶片(Pt-cy)与往年生叶片(Pt-fy)相 比, $L C C$ 和 $\mathrm{N}: \mathrm{P}$ 较小, 而 $L N C$ 和 $L P C$ 较大, 其中 $L P C$ 对氮添加响应显著, 而往年生叶 $L P C$ 无显著变化(图 2)。

\section{3 氮添加对不同生活型植物叶片性状的影响比 较}

比较发现, 不同生活型植物叶性状对氮添加的 响应不同(表2)。经氮添加处理, 各生活型植物的 $L T$ 和 $L C C$ 未发生显著变化, 针叶乔木 $L A$ 和 $L D M C$ 变化 显著, 阔叶乔木 $S L A$ 和 $C C$ 变化显著, 4 种生活型植物 的 $L N C$ 均显著升高, 阔叶乔木、灌木及草本的 $L P C$ 也极显著升高, 而灌木、草本植物 $\mathrm{N}: \mathrm{P}$ 极显著下降。 可以看出, 乔木树种对氮添加的响应包括形态结构 指标(SLA、CC等)和 $N 、 P$ 元素含量的变化两个方面, 而灌木及草本的响应则仅仅体现在 $N$ 、 $\mathrm{P}$ 元素含量及 配比的变化上。

\section{4 氮添加对植物叶性状及其相互关系的影响}

Pearson相关性分析表明, 叶性状之间普遍存在 着显著或极显著相关关系(表3)。SLA与 $L T 、 C C$ 、 $L D M C$ 和 $L C C$ 极显著负相关, 与 $L A 、 L N C 、 L P C$ 和 $\mathrm{N}$ :P极显著正相关, 相关系数值 $L T>L N C>L C C>$ $L D M C>L P C>L A>C C>\mathrm{N}: \mathrm{P}$ 。 $\mathrm{N}: \mathrm{P}$ 与 $L T 、 L P C$ 呈极 显著负相关，与 $L A 、 S L A 、 L N C$ 极显著正相关，与CC
显著正相关, 相关系数值表现为 $L P C>L T>S L A$ 、 $L N C>L A>C C$ 。氮添加处理强度 $(N-T r$. $)$ 与 $S L A$ 和 $\mathrm{N}: \mathrm{P}$ 显著负相关，与CC、LNC、LPC显著正相关，相 关系数数值 $L P C>L N C>C C>\mathrm{N}: \mathrm{P}>S L A$; 所有叶 性状与物种(Sp.)均显著相关, 且氮添加处理和物种 对所有叶性状的交互作用 $(\mathrm{N}-\mathrm{Tr} . \times$ Sp.)显著。

对于与构成叶经济谱相关的关联性状 $L N C$ vs. $S L A 、 L P C$ vs. SLA、 $L N C$ vs. $L T$ 和 $L P C$ vs. $L T$ 进行分 析发现, 氮添加强度增大时, $L N C 、 L P C$ vs. SLA、 $L T$ 的拟合线斜率均发生了明显变化, 且 $L P C$ vs. SLA、 $L T$ 的斜率变化幅度大于 $L N C$ vs. $S L A 、 L T$ 的变化幅度 (图3)。此外, 随着氮添加浓度的增加, LNC:SLA、 $L P C: S L A$ 和 LNC:LT、LPC:LT的值均显著增大(表4), 说明这4组相关关系受氮添加影响显著。

\section{5 氮添加对植物在叶经济谱中位置的影响}

主成分分析结果表明, 第一主成分和第二主成 分的方差解释率分别为 $52.04 \%$ 和 $15.62 \%$, 合计 $67.66 \%$ (表5)。其中主成分 1 主要与 $L N C 、 S L A 、 L C C$ 、 $L P C$ 和 $L A$ 等因子相关，相当于LES理论中的“投资收益”策略轴, 而主成分 2 与 $\mathrm{N}: \mathrm{P} 、 L P C 、 L T$ 等相关度 较高(图4A)。其中，氮添加处理(N-Tr.)对第一主成分 的解释率约为 $-19.9 \%$, 与其他因子相比解释力偏 低; 而对于第二主成分的解释率则高达 $-56.8 \%$, 解 释力仅次于 $\mathrm{N}: \mathrm{P}$ 。这说明 $\mathrm{N}-\mathrm{Tr}$. 促使植物叶性状在第 一、第二主成分上的因子得分均有所下降, 但主要 作用于第二主成分。此外, 进一步计算得到氮添加 在针叶乔木、阔叶乔木、灌木、草本中第一、第二 主成分载荷向量之和的绝对值分别是 $0.77 、 0.73$ 、 0.78 和 0.83 , 说明它对 4 种生活型植物叶片性状的影 响力大小为草本 $>$ 灌木 $>$ 针叶乔木 $>$ 阔叶乔木。

分析11种植物的因子得分情况(图4B), 油松的 第一因子得分最高, 其次是金银忍冬和羊须草, 得

图1 氮添加对叶厚度 $(L T, \mathbf{A})$ 、叶面积 $(L A, \mathbf{B})$ 、比叶面积 $(S L A, \mathbf{C})$ 、叶绿素含量 $(C C, \mathbf{D})$ 和叶干物质含量 $(L D M C, \mathbf{E})$ 的影响(平均 值土标准误差, $n=3)$ 。 Ag, 茶条槭; At, 大火草; Cb, 沙梾; Cc, 羊须草; Cm, 毛榛; Dm, 龙常草; Lm, 金银忍冬; Po, 玉竹; Pt-cy, 油松(当年生叶); Pt-fy, 油松(往年生叶); Qm, 蒙古栎; Ss, 绣线菊。CK, 氮添加浓度为 $0 \mathrm{~kg} \cdot \mathrm{hm}^{-2} \cdot \mathrm{a}^{-1} ; \mathrm{LN}$, 氮添加浓度为50 $\mathrm{kg} \cdot \mathrm{hm}^{-2} \cdot \mathrm{a}^{-1} ; \mathrm{MN}$, 氮添加浓度为 $100 \mathrm{~kg} \cdot \mathrm{hm}^{-2} \cdot \mathrm{a}^{-1} ; \mathrm{HN}$, 氮添加浓度为 $150 \mathrm{~kg} \cdot \mathrm{hm}^{-2} \cdot \mathrm{a}^{-1}$ 。不同小写字母表示氮添加处理间在 $p<$ 0.05水平上差异显著。

Fig. 1 Effects of nitrogen addition on leaf thickness $(L T, \mathbf{A}) 、$ leaf area $(L A, \mathbf{B}) 、$ specific leaf area $(S L A, \mathbf{C})$ 、 chlorophyll content $(C C$, D) and leaf dry matter content $(L D M C, \mathbf{E})($ mean $\pm S E, n=3)$. Ag, Acer ginnala; At, Anemone tomentosa; Cb, Cornus bretchneideri; Cc, Carex callitrichos; Cm, Corylus mandshurica; Dm, Diarrhena mandshurica; Lm, Lonicera maackii; Po, Polygonatum odoratum; Pt-cy, Pinus tabuliformis (current-year leaves); Pt-fy, P. tabuliformis (former-year leaves); Qm, Quercus mongolica; Ss, Spiraea salicifolia. CK, $0 \mathrm{~kg} \cdot \mathrm{hm}^{-2} \cdot \mathrm{a}^{-1}$ nitrogen; LN, $50 \mathrm{~kg} \cdot \mathrm{hm}^{-2} \cdot \mathrm{a}^{-1}$ nitrogen; MN, $100 \mathrm{~kg} \cdot \mathrm{hm}^{-2} \cdot \mathrm{a}^{-1}$ nitrogen; HN, $150 \mathrm{~kg} \cdot \mathrm{hm}{ }^{-2} \cdot \mathrm{a}^{-1}$ nitrogen. Different lowercase letters indicate the significant difference at $5 \%$ level between nitrogen treatments. 

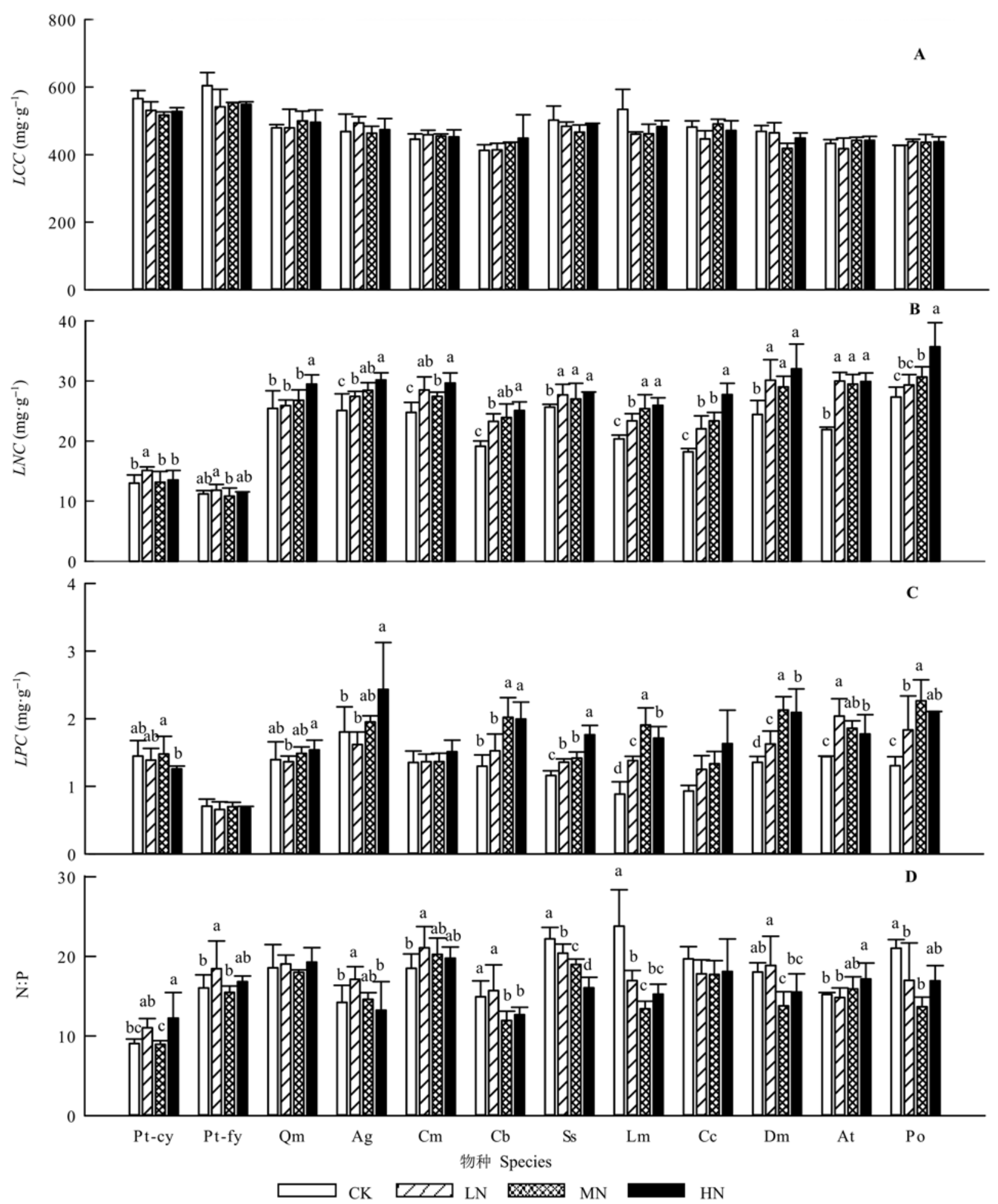

图2 氮添加对叶碳含量 $(L C C, \mathbf{A})$ 、叶氮含量 $(L N C, \mathbf{B})$ 、叶磷含量 $(L P C, \mathbf{C})$ 及氮磷比 $(\mathrm{N}: \mathrm{P}, \mathbf{D})$ 的影响 (平均值土标准误差, $n=3)$ 。 $\mathrm{Ag}$, 茶条械; At, 大火草; Cb, 沙梾; Cc, 羊须草; Cm, 毛榛; Dm, 龙常草; Lm, 金银忍冬; Po, 玉竹; Pt-cy, 油松(当年生叶); $\mathrm{Pt}-\mathrm{fy}$, 油松(往年生叶); Qm, 蒙古係; Ss, 绣线菊。CK, 氮添加浓度为 $0 \mathrm{~kg} \cdot \mathrm{hm}^{-2} \cdot \mathrm{a}^{-1} ; \mathrm{LN}$, 氮添加浓度为 $50 \mathrm{~kg} \cdot \mathrm{hm}^{-2} \cdot \mathrm{a}^{-1} ; \mathrm{MN}$, 氮 添加浓度为 $100 \mathrm{~kg} \cdot \mathrm{hm}^{-2} \cdot \mathrm{a}^{-1} ; \mathrm{HN}$, 氮添加浓度为 $150 \mathrm{~kg} \cdot \mathrm{hm}^{-2} \cdot \mathrm{a}^{-1}$ 。不同小写字母表示氮添加处理间在 $p<0.05$ 水平上差异显著。 Fig. 2 Effects of nitrogen addition on leaf carbon content $(L C C, \mathbf{A})$, leaf nitrogen content $(L N C, \mathbf{B})$, leaf phosphorus content ( $L P C$, C), and N-P ratio (N:P, D)(mean $\pm S E, n=3)$. Ag, Acer ginnala; At, Anemone tomentosa; Cb, Cornus bretchneideri; Cc, Carex callitrichos; Cm, Corylus mandshurica; Dm, Diarrhena mandshurica; Lm, Lonicera maackii; Po, Polygonatum odoratum; Pt-cy, Pinus tabuliformis (current-year leaves); Pt-fy, P. tabuliformis (former-year leaves); Qm, Quercus mongolica; Ss, Spiraea salicifolia. CK, 0 $\mathrm{kg} \cdot \mathrm{hm}^{-2} \cdot \mathrm{a}^{-1}$ nitrogen; LN, $50 \mathrm{~kg} \cdot \mathrm{hm}^{-2} \cdot \mathrm{a}^{-1}$ nitrogen; MN, $100 \mathrm{~kg} \cdot \mathrm{hm}^{-2} \cdot \mathrm{a}^{-1}$ nitrogen; $\mathrm{HN}, 150 \mathrm{~kg} \cdot \mathrm{hm}^{-2} \cdot \mathrm{a}^{-1}$ nitrogen. Different lowercase letters indicate the significant difference at $5 \%$ level between nitrogen treatments. 
表2＼cjkstart不同生活型植物叶片性状对氮添加的响应(平均值土标准误差)

Table 2 Variation of leaf traits in different life forms under different levels of nitrogen addition (mean $\pm S E$ )

\begin{tabular}{|c|c|c|c|c|c|}
\hline \multirow{2}{*}{$\begin{array}{l}\text { 叶性状 } \\
\text { Leaf trait }\end{array}$} & \multirow{2}{*}{$\begin{array}{c}\text { 处理 } \\
\text { Treatment }\end{array}$} & \multicolumn{4}{|c|}{ 生活型 Life form } \\
\hline & & 针叶乔木 Coniferous tree & 润叶乔木 Broadleaf tree & 灌木 Shrub & 草本 Herb \\
\hline 叶厚度 & CK & $716.23 \pm 15.16^{\mathrm{a}}$ & $160.45 \pm 10.44^{\mathrm{a}}$ & $167.45 \pm 18.16^{\mathrm{a}}$ & $199.43 \pm 28.20^{\mathrm{a}}$ \\
\hline \multirow{3}{*}{ Leaf thickness $(\mu \mathrm{m})$} & $\mathrm{LN}$ & $675.67 \pm 27.71^{\mathrm{a}}$ & $136.88 \pm 10.45^{\mathrm{a}}$ & $166.78 \pm 13.89^{\mathrm{a}}$ & $198.07 \pm 23.30^{\mathrm{a}}$ \\
\hline & MN & $689.33 \pm 27.24^{\mathrm{a}}$ & $147.48 \pm 5.23^{\mathrm{a}}$ & $152.16 \pm 7.41^{\mathrm{a}}$ & $168.19 \pm 20.76^{\mathrm{a}}$ \\
\hline & $\mathrm{HN}$ & $661.00 \pm 17.85^{\mathrm{a}}$ & $154.85 \pm 9.76^{\mathrm{a}}$ & $167.69 \pm 16.35^{\mathrm{a}}$ & $161.35 \pm 17.11^{\mathrm{a}}$ \\
\hline 叶面积 & CK & $6.59 \pm 0.14^{\mathrm{ab}}$ & $22.91 \pm 2.70^{\mathrm{a}}$ & $25.59 \pm 5.56^{\mathrm{a}}$ & $39.75 \pm 12.70^{\mathrm{a}}$ \\
\hline \multirow{3}{*}{ Leaf area $\left(\mathrm{cm}^{2}\right)$} & $\mathrm{LN}$ & $7.13 \pm 0.33^{\mathrm{a}}$ & $23.92 \pm 4.38^{\mathrm{a}}$ & $23.79 \pm 4.83^{\mathrm{a}}$ & $39.36 \pm 12.85^{\mathrm{a}}$ \\
\hline & MN & $7.18 \pm 0.01^{\mathrm{a}}$ & $29.33 \pm 4.91^{\mathrm{a}}$ & $25.97 \pm 5.02^{\mathrm{a}}$ & $44.70 \pm 13.22^{\mathrm{a}}$ \\
\hline & $\mathrm{HN}$ & $6.45 \pm 0.12^{b}$ & $26.49 \pm 3.74^{\mathrm{a}}$ & $30.02 \pm 5.96^{\mathrm{a}}$ & $51.44 \pm 20.18^{\mathrm{a}}$ \\
\hline 比叶面积 & CK & $12.42 \pm 0.53^{\mathrm{a}}$ & $24.32 \pm 1.36^{\mathrm{ab}}$ & $36.06 \pm 0.86^{\mathrm{a}}$ & $34.22 \pm 1.12^{\mathrm{a}}$ \\
\hline Specific leaf area & LN & $12.62 \pm 0.33^{\mathrm{a}}$ & $25.95 \pm 0.91^{\mathrm{a}}$ & $35.15 \pm 1.27^{\mathrm{a}}$ & $33.30 \pm 0.92^{\mathrm{a}}$ \\
\hline \multirow{2}{*}{$\left(\mathrm{m}^{2} \cdot \mathrm{kg}^{-1}\right)$} & MN & $11.54 \pm 0.40^{\mathrm{a}}$ & $22.19 \pm 1.43^{a b}$ & $32.66 \pm 1.69^{\mathrm{a}}$ & $32.98 \pm 1.16^{\mathrm{a}}$ \\
\hline & $\mathrm{HN}$ & $12.67 \pm 0.79^{\mathrm{a}}$ & $21.73 \pm 1.19^{b}$ & $32.00 \pm 1.56^{\mathrm{a}}$ & $31.60 \pm 1.09^{\mathrm{a}}$ \\
\hline 叶绿素含量 & CK & $45.73 \pm 1.36^{\mathrm{a}}$ & $43.78 \pm 1.91^{b}$ & $35.20 \pm 1.46^{\mathrm{a}}$ & $30.78 \pm 1.50^{\mathrm{a}}$ \\
\hline Chlorophyll content & LN & $40.37 \pm 1.34^{\mathrm{a}}$ & $46.87 \pm 2.17^{\mathrm{ab}}$ & $37.03 \pm 1.60^{\mathrm{a}}$ & $31.81 \pm 1.74^{\mathrm{a}}$ \\
\hline \multirow[t]{2}{*}{ (SPAD) } & MN & $44.03 \pm 1.95^{\mathrm{a}}$ & $48.75 \pm 1.82^{\mathrm{ab}}$ & $39.65 \pm 1.83^{\mathrm{a}}$ & $34.86 \pm 1.47^{\mathrm{a}}$ \\
\hline & $\mathrm{HN}$ & $42.23 \pm 2.87^{\mathrm{a}}$ & $49.47 \pm 1.21^{\mathrm{a}}$ & $39.71 \pm 1.83^{\mathrm{a}}$ & $33.62 \pm 1.43^{\mathrm{a}}$ \\
\hline 叶干物质含量 & CK & $391.87 \pm 5.43^{b}$ & $381.12 \pm 15.37^{\mathrm{a}}$ & $284.66 \pm 12.41^{\mathrm{a}}$ & $236.01 \pm 16.81^{\mathrm{a}}$ \\
\hline \multirow{3}{*}{$\begin{array}{l}\text { Leaf dry matter content } \\
\left(\mathrm{g} \cdot \mathrm{kg}^{-1}\right)\end{array}$} & $\mathrm{LN}$ & $421.10 \pm 7.77^{\mathrm{a}}$ & $377.22 \pm 3.14^{\mathrm{a}}$ & $287.51 \pm 11.45^{\mathrm{a}}$ & $241.20 \pm 16.62^{\mathrm{a}}$ \\
\hline & MN & $420.03 \pm 3.91^{\mathrm{a}}$ & $395.85 \pm 11.58^{\mathrm{a}}$ & $290.32 \pm 15.77^{\mathrm{a}}$ & $213.13 \pm 23.40^{\mathrm{a}}$ \\
\hline & $\mathrm{HN}$ & $416.17 \pm 7.31^{\mathrm{a}}$ & $384.52 \pm 5.94^{\mathrm{a}}$ & $295.34 \pm 11.52^{\mathrm{a}}$ & $249.49 \pm 11.31^{\mathrm{a}}$ \\
\hline 叶有机碳含量 & CK & $587.59 \pm 10.05^{\mathrm{a}}$ & $473.87 \pm 15.47^{\mathrm{a}}$ & $484.68 \pm 17.84^{\mathrm{a}}$ & $452.51 \pm 7.97^{\mathrm{a}}$ \\
\hline \multirow{3}{*}{$\begin{array}{l}\text { Leaf carbon content } \\
\left(\mathrm{mg} \cdot \mathrm{g}^{-1}\right)\end{array}$} & $\mathrm{LN}$ & $541.67 \pm 27.75^{\mathrm{a}}$ & $486.62 \pm 17.09^{\mathrm{a}}$ & $450.92 \pm 7.49^{\mathrm{a}}$ & $441.45 \pm 8.30^{\mathrm{a}}$ \\
\hline & MN & $553.05 \pm 1.01^{\mathrm{a}}$ & $481.64 \pm 13.02^{\mathrm{a}}$ & $454.37 \pm 6.12^{\mathrm{a}}$ & $449.41 \pm 9.61^{\mathrm{a}}$ \\
\hline & $\mathrm{HN}$ & $548.83 \pm 4.88^{\mathrm{a}}$ & $487.60 \pm 13.23^{\mathrm{a}}$ & $468.28 \pm 11.67^{\mathrm{a}}$ & $450.11 \pm 6.43^{\mathrm{a}}$ \\
\hline 叶氮含量 & CK & $12.09 \pm 0.39^{b}$ & $25.25 \pm 0.79^{\mathrm{Cc}}$ & $22.47 \pm 0.61^{\mathrm{Bc}}$ & $22.96 \pm 0.75^{\mathrm{Cc}}$ \\
\hline \multirow{3}{*}{$\begin{array}{l}\text { Leaf nitrogen content } \\
\left(\mathrm{mg} \cdot \mathrm{g}^{-1}\right)\end{array}$} & $\mathrm{LN}$ & $13.48 \pm 0.47^{\mathrm{a}}$ & $26.60 \pm 0.32^{\mathrm{BCb}}$ & $25.63 \pm 0.56^{\mathrm{Ab}}$ & $28.04 \pm 0.80^{\mathrm{Bb}}$ \\
\hline & MN & $11.91 \pm 0.50^{\mathrm{b}}$ & $27.56 \pm 0.47^{\mathrm{Bb}}$ & $25.81 \pm 0.47^{\mathrm{Ab}}$ & $28.20 \pm 0.62^{\mathrm{Bb}}$ \\
\hline & HN & $12.50 \pm 0.38^{\mathrm{ab}}$ & $29.78 \pm 0.37^{\mathrm{Aa}}$ & $26.93 \pm 0.45^{\mathrm{Aa}}$ & $30.47 \pm 0.74^{\mathrm{Aa}}$ \\
\hline 叶磷含量 & CK & $1.08 \pm 0.12^{\mathrm{a}}$ & $1.60 \pm 0.11^{\mathrm{ABb}}$ & $1.17 \pm 0.05^{\mathrm{Cc}}$ & $1.26 \pm 0.04^{\mathrm{BC}}$ \\
\hline \multirow{3}{*}{$\begin{array}{l}\text { Leaf phosphorus content } \\
\left(\mathrm{mg} \cdot \mathrm{g}^{-1}\right)\end{array}$} & $\mathrm{LN}$ & $1.02 \pm 0.10^{\mathrm{a}}$ & $1.48 \pm 0.05^{\mathrm{Bb}}$ & $1.41 \pm 0.03^{\mathrm{Bb}}$ & $1.68 \pm 0.08^{\mathrm{Ab}}$ \\
\hline & MN & $1.06 \pm 0.11^{\mathrm{a}}$ & $1.70 \pm 0.07^{\mathrm{ABab}}$ & $1.71 \pm 0.07^{\mathrm{Aa}}$ & $1.91 \pm 0.08^{\mathrm{Aa}}$ \\
\hline & $\mathrm{HN}$ & $0.98 \pm 0.07^{\mathrm{a}}$ & $1.92 \pm 0.17^{\mathrm{Aa}}$ & $1.75 \pm 0.05^{\mathrm{Aa}}$ & $1.86 \pm 0.08^{\text {Aab }}$ \\
\hline & CK & $12.54 \pm 1.11^{\mathrm{a}}$ & $16.39 \pm 0.96^{\mathrm{a}}$ & $19.86 \pm 0.89^{\mathrm{Aa}}$ & $18.50 \pm 0.50^{\mathrm{Aa}}$ \\
\hline \multirow{3}{*}{$\begin{array}{l}\text { 叶资(矽比 } \\
\text { leaf } \mathrm{N}: \mathrm{P}\end{array}$} & $\mathrm{LN}$ & $14.75 \pm 1.14^{\mathrm{a}}$ & $18.16 \pm 0.46^{\mathrm{a}}$ & $18.47 \pm 0.61^{\mathrm{ABa}}$ & $17.26 \pm 0.66^{\mathrm{ABab}}$ \\
\hline & MN & $12.44 \pm 0.89^{\mathrm{a}}$ & $16.44 \pm 0.52^{\mathrm{a}}$ & $15.80 \pm 0.72^{\mathrm{Bb}}$ & $15.16 \pm 0.44^{\mathrm{BC}}$ \\
\hline & $\mathrm{HN}$ & $14.40 \pm 0.80^{\mathrm{a}}$ & $16.70 \pm 1.08^{\mathrm{a}}$ & $15.77 \pm 0.59^{\mathrm{Bb}}$ & $16.95 \pm 0.59^{\mathrm{ABb}}$ \\
\hline
\end{tabular}

CK, 氮添加浓度为 $0 \mathrm{~kg} \cdot \mathrm{hm}^{-2} \cdot \mathrm{a}^{-1} ; \mathrm{LN}$, 氮添加浓度为 $50 \mathrm{~kg} \cdot \mathrm{hm}^{-2} \cdot \mathrm{a}^{-1} ; \mathrm{MN}$, 氮添加浓度为 $100 \mathrm{~kg} \cdot \mathrm{hm}^{-2} \cdot \mathrm{a}^{-1} ; \mathrm{HN}$, 氮添加浓度为 $150 \mathrm{~kg} \cdot \mathrm{hm}^{-2} \cdot \mathrm{a}^{-1}$ 。不同小写字 母(a, b, c) 和大写字母 $(\mathrm{A}, \mathrm{B}, \mathrm{C})$ 分别表示不同氮添加水平下叶片性状在 $p<0.05$ 水平和 $p<0.01$ 水平上差异显著。差异显著的数据用加粗字体显示。 CK, $0 \mathrm{~kg} \cdot \mathrm{hm}^{-2} \cdot \mathrm{a}^{-1}$ nitrogen; LN, $50 \mathrm{~kg} \cdot \mathrm{hm}^{-2} \cdot \mathrm{a}^{-1}$ nitrogen; MN, $100 \mathrm{~kg} \cdot \mathrm{hm}^{-2} \cdot \mathrm{a}^{-1}$ nitrogen; HN, $150 \mathrm{~kg} \cdot \mathrm{hm}^{-2} \cdot \mathrm{a}^{-1}$ nitrogen. Different capital or lowercase letters indicate the significant difference at $1 \%$ level or $5 \%$ level among treatments. Data with significant difference were in bold.

表3＼cjkstart叶性状间Pearson相关系数及氮添加处理和物种的影响效应分析

Table 3 Pearson correlation coefficients among leaf traits and the effects of N-treatment and species

\begin{tabular}{|c|c|c|c|c|c|c|c|c|c|c|c|c|}
\hline & $L T$ & $L A$ & SLA & $C C$ & $L D M C$ & $L C C$ & $L N C$ & $L P C$ & $\mathrm{~N}: \mathrm{P}$ & N-Tr. & Sp. & Sig. (N-Tr. $\times$ Sp.) \\
\hline$\overline{L T}$ & 1 & & & & & & & & & -0.06 & $-0.50^{* *}$ & 0.00 \\
\hline$L A$ & $-0.17^{* *}$ & 1 & & & & & & & & 0.06 & $0.37^{* *}$ & 0.00 \\
\hline$S L A$ & $-0.73^{* *}$ & $0.42^{* *}$ & 1 & & & & & & & $-0.12^{*}$ & $0.71^{* *}$ & 0.01 \\
\hline CC & 0.10 & $0.25^{* *}$ & $-0.34^{* *}$ & 1 & & & & & & $0.18^{* *}$ & $-0.30^{* *}$ & 0.00 \\
\hline$L D M C$ & 0.11 & $-0.43^{* *}$ & $-0.56^{* *}$ & $0.35^{* *}$ & 1 & & & & & 0.04 & $-0.72^{* *}$ & 0.00 \\
\hline$L C C$ & $0.44^{* *}$ & $-0.55^{* *}$ & $-0.64^{* *}$ & $0.22^{* *}$ & $0.52^{* *}$ & 1 & & & & -0.04 & $-0.58^{* *}$ & 0.00 \\
\hline$L N C$ & $-0.73^{* *}$ & $0.56^{* *}$ & $0.71^{* *}$ & $0.13^{*}$ & $-0.37^{* *}$ & $-0.60^{* *}$ & 1 & & & $0.25^{* *}$ & $0.62^{* *}$ & 0.00 \\
\hline$L P C$ & $-0.46^{* *}$ & $0.42^{* *}$ & $0.50^{* *}$ & 0.03 & $-0.40^{* *}$ & $-0.54^{* *}$ & $0.71^{* *}$ & 1 & & $0.34^{* *}$ & $0.39^{* *}$ & 0.00 \\
\hline $\mathrm{N}: \mathrm{P}$ & $-0.36^{* *}$ & $0.17^{* *}$ & $0.29^{* *}$ & $0.14^{*}$ & 0.08 & -0.06 & $0.29^{* *}$ & $-0.41^{* *}$ & 1 & $-0.17^{* *}$ & $0.26^{* *}$ & 0.00 \\
\hline
\end{tabular}

$C C$, 叶绿素含量; $L A$, 叶面积; $L C C$, 叶有机碳含量; $L D M C$, 叶干物质含量; $L N C$, 叶氮含量; $L P C$, 叶磷含量; $L T$, 叶厚度; N:P, 氮磷比; SLA, 比叶面 积。N-Tr., 氮添加处理; Sp., 物种; Sig. (N-Tr. $\times$ Sp.), 氮添加处理与物种交互作用的显著性。分析前对数据进行了 $\log _{10}$ 转化。*, $p<0.05 ; * *, p<0.01$ 。 $C C$, chlorophyll content; $L A$, leaf area; $L C C$, leaf carbon content; $L D M C$, leaf dry matter content; $L N C$, leaf nitrogen content; $L P C$, leaf phosphorus content; $L T$, leaf thickness; N:P, N-P ratio; SLA, specific leaf area. N-Tr., N addition treatment; Sp., Species; Sig. (N-Tr. $\times$ Sp.), the significance of N-Tr. by Sp. interactions.

Data were $\log _{10}$-transformed before the analysis. ${ }^{*}, p<0.05 ; * *, p<0.01$. 

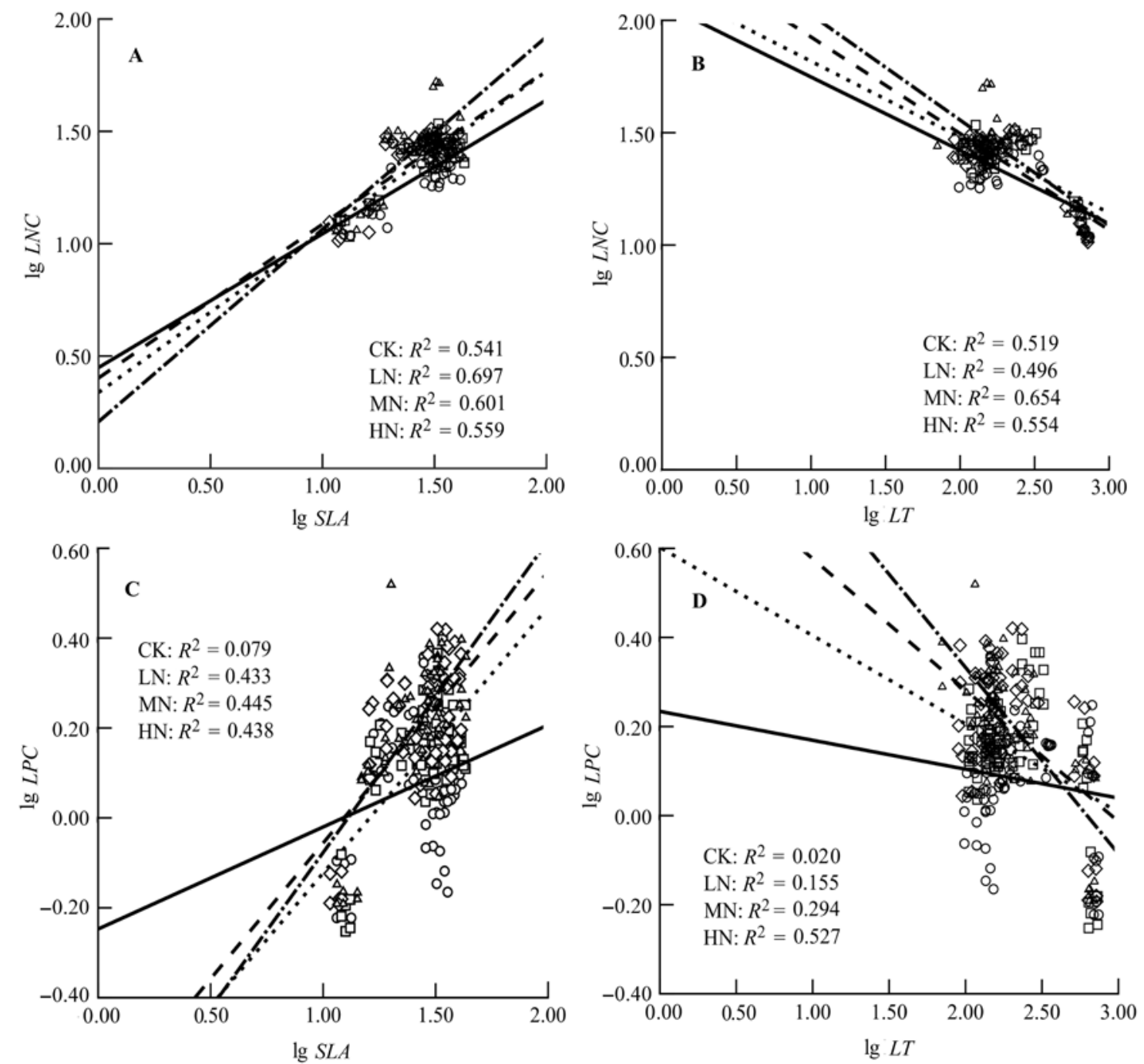

图3 氮添加对叶氮含量 $(L N C)$ 与比叶面积 $(S L A)(\mathbf{A}) 、 L N C$ 与叶厚度 $(L T)(\mathbf{B})$ 、叶磷含量 $(L P C)$ 与 $S L A(\mathbf{C})$ 和 $L P C$ 与 $L T(\mathbf{D})$ 关系的 影响。CK, 氮添加浓度为 $0 \mathrm{~kg} \cdot \mathrm{hm}^{-2} \cdot \mathrm{a}^{-1} ; \mathrm{LN}$, 氮添加浓度为 $50 \mathrm{~kg} \cdot \mathrm{hm}^{-2} \cdot \mathrm{a}^{-1} ; \mathrm{MN}$, 氮添加浓度为 $100 \mathrm{~kg} \cdot \mathrm{hm}^{-2} \cdot \mathrm{a}^{-1} ; \mathrm{HN}$, 氮添加浓 度为 $150 \mathrm{~kg} \cdot \mathrm{hm}^{-2} \cdot \mathrm{a}^{-1}$ 。

Fig. 3 Effects of nitrogen addition on leaf nitrogen content $(L N C)$ vs. specific leaf area (SLA) (A), $L N C$ vs. leaf thickness ( $L T)$ (B), leaf phosphorus content (LPC) vs. SLA (C) and LPC vs. LT (D). CK, $0 \mathrm{~kg} \cdot \mathrm{hm}^{-2} \cdot \mathrm{a}^{-1}$ nitrogen; $\mathrm{LN}, 50 \mathrm{~kg} \cdot \mathrm{hm}^{-2} \cdot \mathrm{a}^{-1}$ nitrogen; MN, 100 $\mathrm{kg} \cdot \mathrm{hm}^{-2} \cdot \mathrm{a}^{-1}$ nitrogen; HN, $150 \mathrm{~kg} \cdot \mathrm{hm}^{-2} \cdot \mathrm{a}^{-1}$ nitrogen.

分最低的是玉竹和大火草, 即油松、金银忍冬和羊 须草具有较高的 $L T 、 L D M C 、 L C C$, 而玉竹和大火 草等具有更大的 $L A 、 S L A 、 L N C$ 和 $L P C$ 。第二因子 得分最高的是金银忍冬、绣线菊和毛榛, 与得分最 低的沙梾和油松当年生叶相比具有更高的 $C C$ 和 $\mathrm{N}: \mathrm{P}$ 。对各生活型植物叶性状在不同氮添加强度下 的因子得分进行比较, 经过氮添加处理的阔叶乔木 (B)、灌木 $(\mathrm{S})$ 及草本 $(\mathrm{H})$ 的位置相比于对照向左下方 发生偏移(第一因子、第二因子得分均降低); 而针叶 乔木(C)的施氮组与对照组相比则向左上方偏移(第
一因子得分下降，而第二因子得分升高) (图4C)。也 就是说, 模拟氮沉降对植物叶片的经济投资策略产 生了一定影响。

\section{3 讨论}

\section{1 氮添加对植物叶片性状的影响}

本试验表明, 氮添加对天然油松林 11 种植物的 叶片性状产生了显著影响: 龙常草、大火草、玉竹 的 $L T$ 和 $S L A$, 蒙古栋、毛榛、绣线菊、大火草和玉 竹 $L A$, 以及油松当年生叶、绣线菊、大火草和玉 
表4 氮添加处理对叶氮含量、叶磷含量与比叶面积、叶厚度比值影响的单因素方差分析(平均值土标准误差, $n=36$ )

Table 4 Results of one-way ANOVA for the effect of nitrogen addition on the ratio of leaf nitrogen content (LNC) over specific leaf area (SLA) and leaf thickness (LT), and leaf phosphorus content ( $L P C$ ) over specific leaf area (SLA) and leaf thickness ( $L T$ ) (mean $\pm S E, n=36$ )

\begin{tabular}{ccccc}
\hline 处理 & 叶氮含量/比叶面积 & 叶磷含量/比叶面积 & \multicolumn{2}{c}{ 叶氮含量/叶厚度 } \\
Treatment & $L N C: S L A$ & $L P C: S L A$ & 叶磷含量/叶厚度 & LNC:LT \\
\hline CK & $0.74 \pm 0.022^{\mathrm{d}}$ & $0.05 \pm 0.002^{\mathrm{b}}: L T$ & $0.12 \pm 0.007^{\mathrm{c}}$ & $0.01 \pm 0.000^{\mathrm{b}}$ \\
LN & $0.85 \pm 0.018^{\mathrm{c}}$ & $0.05 \pm 0.002^{\mathrm{b}}$ & $0.15 \pm 0.009^{\mathrm{bc}}$ & $0.01 \pm 0.001^{\mathrm{b}}$ \\
MN & $0.92 \pm 0.027^{\mathrm{b}}$ & $0.06 \pm 0.002^{\mathrm{a}}$ & $0.16 \pm 0.009^{\mathrm{ab}}$ & $0.01 \pm 0.001^{\mathrm{a}}$ \\
HN & $1.05 \pm 0.033^{\mathrm{a}}$ & $0.07 \pm 0.003^{\mathrm{a}}$ & $0.18 \pm 0.012^{\mathrm{a}}$ & $0.01 \pm 0.001^{\mathrm{a}}$ \\
\hline
\end{tabular}

$\mathrm{CK}$, 氮添加浓度为 $0 \mathrm{~kg} \cdot \mathrm{hm}^{-2} \cdot \mathrm{a}^{-1} ; \mathrm{LN}$, 氮添加浓度为 $50 \mathrm{~kg} \cdot \mathrm{hm}^{-2} \cdot \mathrm{a}^{-1} ; \mathrm{MN}$, 氮添加浓度为 $100 \mathrm{~kg} \cdot \mathrm{hm}^{-2} \cdot \mathrm{a}^{-1} ; \mathrm{HN}$, 氮添加浓度为 $150 \mathrm{~kg} \cdot \mathrm{hm}{ }^{-2} \cdot \mathrm{a}^{-1}$ 。不同字母表 示不同氮添加强度下叶片性状差异显著 $(p<0.05)$ 。

$\mathrm{CK}, 0 \mathrm{~kg} \cdot \mathrm{hm}^{-2} \cdot \mathrm{a}^{-1}$ nitrogen; LN, $50 \mathrm{~kg} \cdot \mathrm{hm}^{-2} \cdot \mathrm{a}^{-1}$ nitrogen; MN, $100 \mathrm{~kg} \cdot \mathrm{hm}^{-2} \cdot \mathrm{a}^{-1}$ nitrogen; $\mathrm{HN}, 150 \mathrm{~kg} \cdot \mathrm{hm}^{-2} \cdot \mathrm{a}^{-1}$ nitrogen. Different lowercase letters indicate the significant difference at $5 \%$ level among treatments.

表5 叶性状在主成分分析中的载荷

Table 5 Loadings of leaf traits in principal components analyses

\begin{tabular}{|c|c|c|c|c|c|c|c|c|c|c|}
\hline $\begin{array}{l}\text { 载荷 } \\
\text { Loading }\end{array}$ & $\begin{array}{c}\text { 叶厚度 } \\
\text { Leaf } \\
\text { thickness }\end{array}$ & $\begin{array}{c}\text { 叶面积 } \\
\text { Leaf area }\end{array}$ & $\begin{array}{c}\text { 比叶面积 } \\
\text { Specific leaf } \\
\text { area }\end{array}$ & $\begin{array}{c}\text { 叶绿素含量 } \\
\text { Chlorophyll } \\
\text { content }\end{array}$ & $\begin{array}{c}\text { 叶干物质含量 } \\
\text { Leaf dry matter } \\
\text { content }\end{array}$ & $\begin{array}{c}\text { 叶碳含量 } \\
\text { Leaf carbon } \\
\text { content }\end{array}$ & $\begin{array}{c}\text { 叶氮含量 } \\
\text { Leaf nitrogen } \\
\text { content }\end{array}$ & $\begin{array}{c}\text { 叶磷含量 } \\
\text { Leaf phosphorus } \\
\text { content }\end{array}$ & $\begin{array}{c}\text { 氮磷比 } \\
\text { N-P ratio }\end{array}$ & $\begin{array}{c}\text { 氮添加处理 } \\
\mathrm{N} \text { addition } \\
\text { treatment }\end{array}$ \\
\hline $\begin{array}{l}\text { 主成分1 } \\
\text { Principal } \\
\text { component } 1\end{array}$ & 0.70 & -0.64 & -0.83 & 0.09 & 0.58 & 0.80 & -0.91 & -0.80 & -0.11 & -0.20 \\
\hline $\begin{array}{l}\text { 主成分2 } \\
\text { Principal } \\
\text { component } 2\end{array}$ & -0.40 & 0.08 & 0.16 & 0.32 & 0.33 & 0.10 & 0.24 & -0.43 & 0.94 & -0.57 \\
\hline
\end{tabular}

两个主成分总的解释力为 $67.66 \%$, 其中主成分 1 和主成分 2 解释力分别为 $52.04 \%$ 和 $15.62 \%$ 。

The total explanation of two principal component is $67.66 \%$, the explanation of principal component 1 and component 2 are $52.04 \%$ and $15.62 \%$, respectively.

竹的 $L D M C$ 都产生了显著性差异, 9种植物叶片的 $\mathrm{N}: P$ 随氮添加浓度发生显著变化, 全部植物的 $L N C$ 与大多数植物的 $C C 、 L P C$ 显著升高。

一般认为, 氮添加会导致 $S L A$ 的增大(Rose et al., 2013), 因为氮添加促进了植物的生长而提高了 植物的光合效率(Ordoñez et al., 2009)。万宏伟等 (2008)和黄菊䒯等(2009)研究发现, 羊草(Leymus chinensis)等几种草本植物的SLA因氮添加而显著增 大; Bassin等(2009)的氮添加实验也表明, 虽然大多 数植物叶片的比叶重 (LMA, SLA 的倒数)无显著变 化, 但两种植物叶片的 $L M A$ 显著降低(即SLA显著增 大)。然而, 并非所有研究结果都是如此, 赵新风等 (2014)发现, 钠猪毛菜(Salsola nitraria)受氮添加影 响, SLA 呈下降趋势, $L D M C$ 呈增大趋势, 与上述结 果相反。研究表明, 较小的SLA和较高的 $L D M C$ 使得 植物具有更好的资源获取能力、碳氮固持效率及水 分利用效率, 在逆境中更具优势(Wilson et al., 1999; Aerts \& Chapin, 2000; Zhang et al., 2007)。因此, 这 两项指标常被用来指示植物对环境变化的响应策 略, SLA的减小和 $L D M C$ 的增大往往意味着环境条件 的恶化, 因为植物开始趋向于保守的生长策略 (Rose et al., 2013)。本研究结果表明, 大部分植物的 $S L A$ 和 $L D M C$ 并没有表现出显著差异, 只有茶条枫
和玉竹的 $S L A$ 呈显著减小趋势, 油松当年生叶和玉 竹叶片的 $L D M C$ 显著增大, 与赵新风等(2014)的结 果相一致。这说明不同植物对氮添加的响应不同。 并且, 氮添加对油松、茶条枫和玉竹这 3 种植物的生 长是不利的。

另外, 本研究中 11 种植物叶片的 $C C$ 和 $L N C$ 增 大、 $L C C$ 保持不变的趋势与前人研究结果一致, 但 多数植物 $L P C$ 显著上升、部分植物叶片 $\mathrm{N}: \mathrm{P}$ 显著下降 的变化趋势却与其他研究中 $L P C$ 降低而 $\mathrm{N}: \mathrm{P}$ 上升的 结果相反(李德军等, 2005; Rose et al., 2013; 刘洋 等, 2013)。有研究表明, 在N供应受限制的环境中, 氮添加会提高土壤中活性氮的含量, 缓解 $\mathrm{N}$ 的限制 作用, 促进植物的生长, 并使 $\mathrm{N}: \mathrm{P}$ 显著增大、P逐渐 短缺(陈凌云, 2010; 刘洋等, 2013)。遆超普和颜晓元 (2010)提供的数据显示, 山西的铵态氮、硝态氮沉降 量与排放值的比值分别为 1.58 和 0.26 , 氮湿沉降总 量由 1980 年的 $0.20 \mathrm{Tg} \cdot \mathrm{a}^{-1}$ 增长到 2007 年的 0.47 $\mathrm{Tg} \cdot \mathrm{a}^{-1}$, 超过全国平均水平, 并且山西是全国氮沉 降增速最快的地区。以本试验中对照组数值做参考, 11 种植物 $L N C$ 的平均值为 $21.37 \mathrm{mg} \cdot \mathrm{g}^{-1}$, 略高于全球 和全国陆生植物的平均水平(分别为 20.1 和 20.2 $\left.\mathrm{mg} \cdot \mathrm{g}^{-1}\right) ; L P C$ 的平均值为 $1.26 \mathrm{mg} \cdot \mathrm{g}^{-1}$, 低于全球、全 国水平(分别为 1.8 和 $1.46 \mathrm{mg} \cdot \mathrm{g}^{-1}$ ); $\mathrm{N}: \mathrm{P}$ 的均值为17.6, 

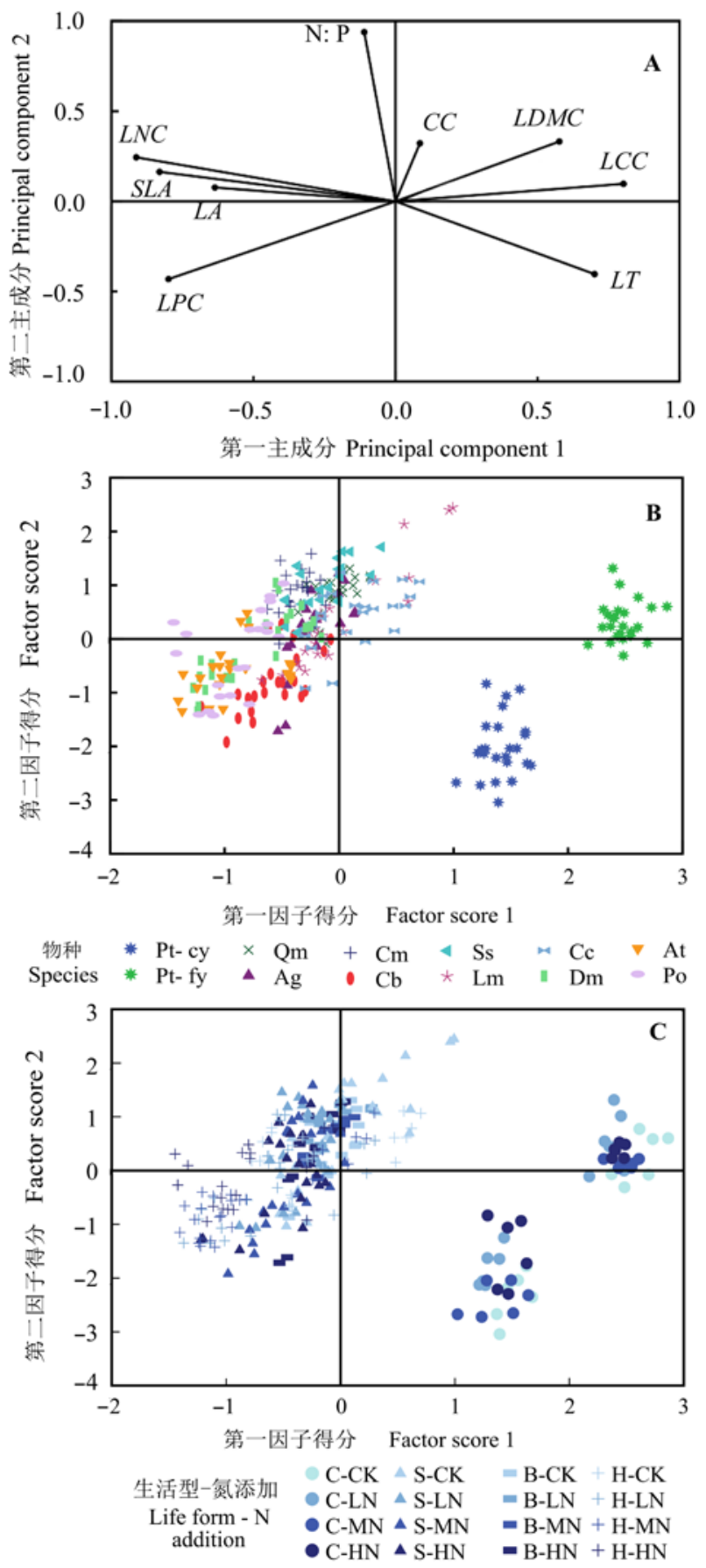

明显高于全球陆生植物的平均水平13.8及全国平均 水平16.3 (Reich \& Oleksyn, 2004; Han et al., 2005)。 以叶片 $\mathrm{N}: \mathrm{P}$ 作为判断植物生长限制因子的指标, 其 中 $\mathrm{N}: \mathrm{P}<14$ 表明氮限制、N:P $>16$ 代表磷限制、处于 二者之间则为氮磷共同限制或者均不受限(Aerts \& Chapin, 2000; Güsewell, 2004), 相对于全球和全国 而言, 山西的植物表现为较明显的磷元素缺乏, 而 非受到氮素限制。因此, 施加过量的氮素可能不会 像其他在氮素缺乏地区进行的实验一样起到促进植 物生长的作用, 还可能不利于植物的生长(这与SLA
图4 天然油松林主要植物的叶性状主成分分析结果: 天然 油松林主要植物的叶性状载荷 $(\mathbf{A})$ 、因子得分 $(\mathbf{B})$ 及对氮添加 的响应 $(\mathbf{C})$ 。分析前对数据进行了 $\log 10$ 转化。CC, 叶绿素含 量; $L A$, 叶面积; $L C C$, 叶有机碳含量; $L D M C$, 叶干物质含 量; $L N C$, 叶氮含量; $L P C$, 叶磷含量; $L T$, 叶厚度; $\mathrm{N}: \mathrm{P}$, 氮磷 比; SLA，比叶面积。Ag, 茶条槭; At, 大火草; Cb, 沙梾; Cc, 羊须草; Cm, 毛榛; Dm, 龙常草; Lm, 金银忍冬; Po, 玉竹; Pt-cy, 油松(当年生叶); Pt-fy, 油松(往年生叶); Qm, 蒙古柇; $\mathrm{Ss}$, 绣线菊。 $\mathrm{C}$, 针叶乔木; $\mathrm{B}$, 阔叶乔木; $\mathrm{H}$, 草本; $\mathrm{S}$, 灌木。 $\mathrm{CK}$, 氮添加浓度为 $0 \mathrm{~kg} \cdot \mathrm{hm}^{-2} \cdot \mathrm{a}^{-1} ; \mathrm{LN}$, 氮添加浓度为 50 $\mathrm{kg} \cdot \mathrm{hm}^{-2} \cdot \mathrm{a}^{-1} ; \mathrm{MN}$, 氮添加浓度为 $100 \mathrm{~kg} \cdot \mathrm{hm}^{-2} \cdot \mathrm{a}^{-1} ; \mathrm{HN}$, 氮添 加浓度为 $150 \mathrm{~kg} \cdot \mathrm{hm}^{-2} \cdot \mathrm{a}^{-1}$ 。

Fig. 4 Results of principle component analyses (PCA) of leaf traits across common species in the natural Pinus tabuliformis forest. Loadings of leaf traits across primary species in the natural forest of $P$. tabuliformis (A), factor scores of different species (B) and responses to nitrogen addition (C). Data were $\log _{10}$-transformed before analysis. $C C$, chlorophyll content; $L A$, leaf area; $L C C$, leaf carbon content; $L D M C$, leaf dry matter content; $L N C$, leaf nitrogen content; $L P C$, leaf phosphorus content; $L T$, leaf thickness; N:P, N-P ratio; SLA, specific leaf area. Ag, Acer ginnala; At, Anemone tomentosa; Cb, Cornus bretchneideri; Cc, Carex callitrichos; Cm, Corylus mandshurica; Dm, Diarrhena mandshurica; Lm, Lonicera maackii; Po, Polygonatum odoratum; Pt-cy, Pinus tabuliformis (current-year leaves); Pt-fy, P. tabuliformis (former-year leaves); Qm, Quercus mongolica; Ss, Spiraea salicifolia. C, coniferous tree; B, broadleaf tree; $\mathrm{H}$, herb; $\mathrm{S}$, shrub. CK, $0 \mathrm{~kg} \cdot \mathrm{hm}^{-2} \cdot \mathrm{a}^{-1}$ nitrogen; $\mathrm{LN}, 50 \mathrm{~kg} \cdot \mathrm{hm}^{-2} \cdot \mathrm{a}^{-1}$ nitrogen; MN, $100 \mathrm{~kg} \cdot \mathrm{hm}^{-2} \cdot \mathrm{a}^{-1}$ nitrogen; $\mathrm{HN}, 150 \mathrm{~kg} \cdot \mathrm{hm}^{-2} \cdot \mathrm{a}^{-1}$ nitrogen.

和 $L D M C$ 预测的结果一样)。目前, 氮添加导致植物 叶片 $L P C$ 显著上升和 $\mathrm{N}: \mathrm{P}$ 显著下降的类似研究结果 还未见报道。据推测, 这种现象的出现可能与本试 验地区土壤氮素供应充足而磷供应缺乏的自然条 件、较高的施氮水平, 以及研究对象本身的物种特 性不同于其他实验有关。而关于叶片 $\mathrm{N}: \mathrm{P}$ 显著下降 的罕见趋势, 可以从 $\mathrm{N}: \mathrm{P}$ 与 $L N C 、 L P C$ 相关关系的研 究中找到答案。大量研究表明, 植物 $L P C$ 与 $\mathrm{N}: \mathrm{P}$ 存在 显著的负相关关系，而 $L N C$ 与 $\mathrm{N}: \mathrm{P}$ 没有显著的相关 性或相关系数很小(Güsewell, 2004; He et al., 2006, 2008)。本研究中, $\mathrm{N}: P$ 虽然与 $L N C$ 和 $L P C$ 都存在极显 著的相关性, 但前者的相关系数远小于后者。由此 可见, 植物叶片 $\mathrm{N}: \mathrm{P}$ 主要受 $\mathrm{P}$ 含量的影响。因此, 虽 然绝大部分植物的 $L N C$ 和 $L P C$ 受到氮添加的影响均 显著上升，但其中5种植物叶片的 N:P因主要受到 $L P C$ 升高的影响而显著下降。

\section{2 植物叶片应对环境变化的资源权衡策略}

黄海侠等(2013)发现，同种植物叶片的物质分 配策略在异龄叶间存在显著差异: 当年生叶的SLA 显著大于往年生叶、 $L D M C$ 比往年生叶小, 且当年 
生叶性状的变异系数往往高于往年生叶。本研究也 从油松当年生叶和往年生叶的性状比较中, 发现了 类似的结果。这是因为叶片在生长初期往往需要较 多的资源来优化同化器官、最大程度地获取光资源 (Sultan, 2000); 但在老化过程中, 内部养分元素(如 氮和磷)发生迁移且光合速率降低(Bleecker, 1998; Ono et al., 2001), 结构充分形成并固化, 对环境变 化的响应就变得相对迟缓(Oguchi et al., 2003); 老 叶为忍受多年环境变化, 在机械和防御构造上的额 外投资较多(Chabot \& David, 1982)。此外, 新叶对 环境变化敏感, 轻微的环境扰动就能诱导其性状发 生改变, 表现出较强的可塑性(Wyka et al., 2000; Frak et al., 2001)。因此, 叶片的物质投资和分配格 局是随叶龄变化的, 这是植物适应环境的一个重要 途径(Silvertown et al., 1997)。

通常, 具有较高SLA、LNC和 $L P C$ 的叶片, 往往 具有较高的光合速率和生长速率, 并且更薄、更可 口、更易啃食, 寿命也更短, 具有更大的 $L T$ 和 $L D M C$ 的植物则相反(Crawley, 1983; Kikuzawa, 1991)。大 量研究表明, 代表光能利用效率的SLA (Westoby et al., 2002)与代表光合速率的LNC (Grime, 2001)及间 接反映生长速率的 $\mathrm{N}: \mathrm{P}$ 呈极显著正相关关系, 而与 代表物理支撑结构的 $L T 、 L D M C$ 和 $L C C$ 呈显著负相 关关系(Wilson et al., 1999)。这是因为植物在特定的 环境条件下可利用的资源总量是有限的, 从养分配 置角度讲, 若一方面的资源投入大, 则其他方面的 投入就要减少, 从而使植物性状之间保持普遍的协 同变化关系(Shipley et al., 2006)。本研究结果与上述 结论相一致, $S L A$ 与 $L T 、 C C 、 L D M C$ 和 $L C C$ 极显著负 相关，与 $L A 、 L N C 、 L P C$ 和 $\mathrm{N}: \mathrm{P}$ 极显著正相关，作为 一个核心性状将所有性状联系起来, 体现出叶片在 功能效率和结构建成两个方面 “此消彼长”的资源权 衡投资策略(Wright et al., 2005; Ordoñez et al., 2009)。从各种植物在主成分分析(PCA)第一主成分 轴上的分布情况来看, 得分最高的油松和得分最低 的玉竹、大火草, 分别具有叶厚、光合能力弱、生 长缓慢、寿命长和叶薄、光合能力强、生长快速、 寿命短的特点, 恰好反映出两种完全不同的生存策 略, 验证了LES理论(陈荣婷和许振柱, 2014)。此外, $L N C$ 和 $L P C$ 与 $S L A$ 和 $L T$ 之间相关拟合曲线的斜率在 氮添加影响下均显著增大。类似地, Fonseca等(2000) 发现SLA和 $L A$ 的关系在生境内部和不同生境之间都
不相同; Wright等(2001)发现SLA与 $L N C$ 的相关关系 在不同水位处理和氮素处理下都有显著差异; Pensa 等(2010)也发现，同种植物 LNC 与叶寿命的关系随 着纬度梯度不断增长。这说明, 植物叶片性状之间 的相关关系并非一成不变, 而是随生境变化而变化 的。因此, 我们在试图利用叶性状普遍存在的相关 关系, 由部分易获取的功能性状来预测其他相对难 以测量的性状值及变化趋势时, 需要综合考虑局域 气候-地理特点或者综合的植物功能型属性。

PCA结果表明, 植物在多元特征空间中的位置 在氮添加的作用下向左侧发生了较为明显的横向迁 移(图4C), 即趋向于叶片更大、更薄、生长更快和 寿命更短的“快速投资-收益型”策略。而在垂直方向 上, 阔叶乔木、灌木和草本植物均朝下移动, 针叶乔 木则向上移动, 说明不同植物对于同一环境因素的 变化有不同的响应策略, 即不同植物叶片特征对环 境变化的响应是异质的(Donovan et al., 2011; Wright \& Sutton-Grier, 2012)。Wright等(2004)指出, 全球尺度上的温度和降水因子对植物的影响总体上 是十分微弱的, 植物通过不断调节自身的功能性状 及其权衡关系, 稳固地占据着一定的生态位空间, 从而部分抵消了温度和降水变化所带来的影响。在 本研究中, 虽然 11 种植物在特征空间中都发生了明 显的位移, 但物种之间的相对位置保持不变, 在群 落水平上维持了叶片经济的内稳态。并且, 在综合 各项性状的大幅度变异之后, 植物在第一主轴(即 LES)上的位移仅为 -0.20 , 说明在局域范围内, 氮添 加对植物生存策略的影响也非常有限。也就是说, 尽管局域种库内植物对环境的响应是多样化的, 但 多种多样的性状变异并不足以改变植物在第一主轴 上的相对位置, 也没有大幅度改变整个群落在第一 主轴上的位置, 植物依旧维持着相当稳定的生态 位。因此, 无论是在全球尺度还是局域尺度上, 当环 境发生变化时, 植物将自发改变生活策略, 发挥权 衡作用对资源进行重新调配, 在很大程度上维持种 间相对位置和群落整体结构的稳定性。这种调节和 适应机制是植物本身所固有的, 并不依赖于环境气 候变化而产生、发展, 也不受环境因素所主导, 故第 一主轴的形成是基于植物根本性的生态学权衡策略 独立演变而来的(Wright et al., 2004, 2005; Shipley et al., 2006; Wright \& Sutton-Grier, 2012)。

综上所述, 氮添加对山西太岳山天然油松林叶 
片性状的影响显著, 不同类型、不同年龄的植物叶 片对氮添加的响应各不相同, 叶性状间的相关性随 氮添加强度而改变, 植物叶经济谱及其涉及的叶性 状权衡关系被证实存在, 植物叶片在氮添加的影响 下更趋向于“快速投资-收益型”策略, 第一主轴的形 成基于植物的生态学权衡策略而不受环境因素主 导。本研究对于揭示植物性状对大气氮沉降等气候 变化的响应与适应机制具有一定意义。

基金项目 中央高校基本科研业务费专项资金(TD2011-07)、国家自然科学基金青年基金(31000263)、 国家自然科学基金面上项目(31470506)。

致谢 感谢中国科学院地理研究所“可桢杰出青年 学者”项目(2013RC102)资助。感谢生物多样性与生 态工程教育部重点实验室开放课题资助。感谢山西 太岳山灵空山林场对研究工作的支持。感谢北京林 业大学资源与生态系统过程北京市重点实验室赵秀 海老师对实验方案的指导, 以及秦书琪、马冰倩、 刘宇三位同学在野外工作中给予的帮助。

\section{参考文献}

Aber JD, McDowell W, Nadelhoffer K, Magill A, Berntsen G, Kamakea M, McNulty S, Currie W, Rustad L, Fernandez I (1998). Nitrogen saturation in temperate forest ecosystems: Hypothesis revisited. BioScience, 48, 921-934.

Aber JD, Nadelhoffer KJ, Steudler P, Melillo JM (1989). Nitrogen saturation in northern forest ecosystems. BioScience, 39, 378-386.

Adams MA, Ineson P, Binkley D, Cadisch G, Tokuchi N, Scholes M, Hicks K (2004). Soil functional responses to excess nitrogen inputs at global scales. AMBIO, 33, 530-536.

Aerts R, Chapin SF (2000). The mineral nutrition of wild plants revisited: A re-evaluation of processes and patterns. Advances in Ecological Research, 30, 1-67.

Albert CH, Grasseina F, Schurrd FM, Vieilledente G, Violle C (2011). When and how should intraspedic variabi lity be considered in trait-based plant ecology? Perspectives in Plant Ecology, Evolution, and Systematics, 13, 217-225.

Albert CH, Thuiller W, Yoccoz NG, Douzet R, Aubert S, Lavorel S (2010a). A multi-trait approach reveals the structure and the relative importance of intra- vs. interspecific variability in plant traits. Functional Ecology, 24, 1192-1201.

Albert CH, Thuiller W, Yoccoz NG, Soudant A, Boucher F, Saccone P, Lavorel S (2010b). Intraspecific functional variability: Extent, structure and sources of variation. Journal of Ecology, 98, 603-613.

Bassin S, Werner RA, Sörgel K, Volk M, Buchmann N, Fuhrer $\mathrm{J}$ (2009). Effects of combined ozone and nitrogen deposi- tion on the in situ properties of eleven key plant species of a subalpine pasture. Oecologia, 158, 747-756.

Bleecker AB (1998). The evolutionary basis of leaf senescence: Method to the madness? Current Opinion in Plant Biology, 1, 73-78.

Blonder B, Violle C, Enquist BJ, Cornelissen H (2013). Assessing the causes and scales of the leaf economics spectrum using venation networks in Populus tremuloides. Journal of Ecology, 101, 981-989.

Bobbink R, Hornung M, Roelofs JGM (1998). The effects of air-borne nitrogen pollutants on species diversity in natural and semi-natural European vegetation. Journal of Ecology, 86, 717-738.

Chabot BF, David JH (1982). The ecology of leaf life spans. Annual Review of Ecology and Systematics, 13, 229-259.

Chen LY (2010). Effects of N, P Addition on N:P Stoichiometry of Different Functional Groups in Potentilla fruticosa Community in a Sub-alpine Meadow. Master degree dissertation, Lanzhou University, Lanzhou. (in Chinese with English abstract) [陈凌云 (2010). 添加氮磷对亚高寒草 甸金露梅群落各功能群化学计量学特征的影响. 硕士 学位论文, 兰州大学, 兰州.]

Chen YT, Xu ZZ (2014). Review on research of leaf economics spectrum. Chinese Journal of Plant Ecology, 38, 1135-1153. (in Chinese with English abstract) [陈莹婷, 许振柱 (2014). 植物叶经济谱的研究进展. 植物生态学 报, 38, 1135-1153.]

Clark CM, Tilman D (2008). Loss of plant species after chronic low-level nitrogen deposition to prairie grasslands. Nature, 451, 712-715.

Comelissen JHC, Cerabolini B, Castro-Diez P, Villar-Salvador P, Montserrat-Marti G, Puyravaud JP, Maestro M, Werger MJA, Aerts R (2003). Functional traits of woody plants: Correspondence of species rankings between field adults and laboratory-grown seedlings? Journal of Vegetation Science, 14, 311-322.

Crawley MJ (1983). Herbivory: The Dynamics of Animal-Plant Interactions. Blackwell Scientific Publications, Oxford, UK.

Donovan LA, Maherali H, Caruso CM, Huber H, de Kroon H (2011). The evolution of the worldwide leaf economics spectrum. Trends in Ecology Evolution, 26, 88-95.

Fang YT, Mo JM, Zhou GY, Xue JH (2005). Response of diameter at breast height increase to $\mathrm{N}$ addition in forests of Dinghushan Biosphere Reserve. Journal of Tropical and Subtropical Botany, 13(3), 198-204. (in Chinese with English abstract) [方运霆, 莫江明, 周国逸, 薛璟花 (2005). 鼎湖山主要森林类型植物胸径生长对氮沉降增 加的初期响应. 热带亚热带植物学报, 13(3), 198-204.]

Fenn ME, Poth MA, Johnson DW (1996). Evidence for nitrogen saturation in the San Bernardino Mountains in Southern California. Forest Ecology and Management, 82,

www.plant-ecology.com 
211-230.

Fonseca CR, Overton JM, Collins B, Westoby M (2000). Shifts in trait combinations along rainfall and phosphorus gradients. Journal of Ecology, 88, 964-977.

Frak E, Roux XL, Millard P, Dreyer E, Jaouen G, Saint-Joanis $B$, Wendler R (2001). Changes in total leaf nitrogen and partitioning of leaf nitrogen drive photosynthetic acclimation to light in fully developed walnut leaves. Plant, Cell \& Environment, 24, 1279-1288.

Freschet GT, Cornelissen JHC, van Logtestijn RSP, Aerts R (2010). Evidence of the 'plant economics spectrum' in a subarctic flora. Journal of Ecology, 98, 362-373.

Grime JP (2001). Plant Strategies, Vegetation Processes and Ecosystem Properties. John Wiley \& Sons, Chichester, UK.

Güsewell S (2004). N:P ratios in terrestrial plants: Variation and functional significance. New Phytologist, 164, 243-266.

Han WX, Fang YT, Guo DL, Zhang Y (2005). Leaf nitrogen and phosphorus stoichiometry across 753 terrestrial plant species in China. New Phytologist, 168, 377-385.

He JS, Fang JY, Wang ZH, Guo DL, Flynn DFB, Geng Z (2006). Stoichiometry and large-scale patterns of leaf carbon and nitrogen in the grassland biomes of China. Oecologia, 149, 115-122.

He JS, Han XG (2010). Ecological stoichiometry: Searching for unifying principles from individuals to ecosystems. Chinese Journal of Plant Ecology, 34, 2-6. (in Chinese with English abstract) [贺金生, 韩兴国 (2010). 生态化学计 量学: 探索从个体到生态系统的统一化理论. 植物生态 学报, 34, 2-6.]

He JS, Wang L, Flynn DFB, Wang XP, Ma WH, Fang JY (2008). Leaf nitrogen:phosphorus stoichiometry across Chinese grassland biomes. Oecologia, 155, 301-310.

Holland EA, Dentener FJ, Braswell BH, Sulzman JM (1999). Contemporary and pre-industrial global reactive nitrogen budgets. Biogeochemistry, 46, 7-43.

Huang HX, Yang XD, Sun BW, Zhang ZH, Yan ER (2013). Variability and association of leaf traits between current-year and former-year leaves in evergreen trees in Tiantong, Zhejiang, China. Chinese Journal of Plant Ecology, 37, 912-921. (in Chinese with English abstract) [黄海侠, 杨晓东, 孙宝伟, 张志浩, 阎恩荣 (2013). 浙 江天童常绿植物当年生与往年生叶片性状的变异与关 联. 植物生态学报, 37, 912-921.]

Huang JY, Yuan ZY, Li LH (2009). Changes in N, P and specific leaf area of green leaves of Leymus chinensis along nitrogen, phosphorus and water gradients. Chinese Journal of Plant Ecology, 33, 442-448. (in Chinese with English abstract) [黄菊䒯, 袁志友, 李凌浩 (2009). 羊草绿叶 氮、磷浓度和比叶面积沿氮、磷和水分梯度的变化. 植 物生态学报, 33, 442-448.]
Kikuzawa K (1991). A cost-benefit analysis of leaf habit and leaf longevity of trees and their geographical pattern. The American Naturalist, 138, 1250-1263.

Laughlin DC (2011). Nitrification is linked to dominant leaf traits rather than functional diversity. Journal of Ecology, 99, 1091-1099.

Leishman MR, Haslehurst T, Ares A, Baruch Z (2007). Leaf trait relationships of native and invasive plants: Community- and global-scale comparisons. New Phytologist, 176, 635-643.

Li DJ, Mo JM, Fang YT, Li ZA (2005). Effects of simulated nitrogen deposition on biomass production and allocation in Schima superba and Cryptocarya concinna seedlings in subtropical China. Acta Phytoecologica Sinica, 29, 543-549. (in Chinese with English abstract) [李德军, 莫 江明, 方运霆, 李志安 (2005). 模拟氮沉降对南亚热带 两种乔木幼苗生物量及其分配的影响. 植物生态学报, 29, 543-549.]

Li HS, Wang JS, Liu X, Jiang SS, Zhang CY, Zhao XH (2014). Effects and its sustained effect of simulated nitrogen deposition on soil respiration in Pinus tabulaeformis forests in the Taiyue Mountain, China. Acta Scientiae Circumstantiae, 34, 238-249. (in Chinese with English abstract) [李化 山, 汪金松, 刘星, 蒋思思, 张春雨, 赵秀海 (2014). 模 拟氮沉降对太岳山油松林土壤呼吸的影响及其持续效 应. 环境科学学报, 34, 238-249.]

Li XR, Liu QJ, Cai Z, Ma ZQ (2007). Specific leaf area and leaf area index of conifer plantations in Qianyanzhou Station of subtropical China. Journal of Plant Ecology (Chinese Version), 31, 93-101. (in Chinese with English abstract) [李轩然, 刘琪璟, 蔡哲, 马泽清 (2007). 千烟洲 针叶林的比叶面积及叶面积指数. 植物生态学报, 31, 93-101.]

Liu XJ, Zhang Y, Han WX, Tang AH, Shen JL, Cui ZL, Vitousek P, Erisman JW, Goulding K, Christie P, Fangmeier A, Zhang FS (2013). Enhanced nitrogen deposition over China. Nature, 494, 459-462.

Liu Y, Zhang J, Chen YM, Chen L, Liu Q (2013). Effect of nitrogen and phosphorus fertilization on biomass allocation and C:N:P stoichiometric characteristics of Eucalyptus grandis seedlings. Chinese Journal of Plant Ecology, 37, 933-941. (in Chinese with English abstract) [刘洋, 张 健, 陈亚梅, 陈碟, 刘强 (2013). 氮磷添加对巨桉幼苗 生物量分配和C:N:P化学计量特征的影响. 植物生态学 报, 37, 933-941.]

Magill AH, Aber JD, Currie WS, Nadelhoffer KJ, Martin ME, McDowell WH, Melillo JM, Steudler P (2004). Ecosystem response to 15 years of chronic nitrogen additions at the Harvard Forest LTER, Massachusetts, USA. Forest Ecology and Management, 196, 7-28.

Maire V, Gross N, Hill D, Martin R, Wirth C, Wright IJ, 
Soussana JF (2013). Disentangling coordination among functional traits using an individual-centred model: Impact on plant performance at intra- and inter-specific levels. PLOS ONE, 8, e77372. doi: 10.1371/journal.pone.0077372.eCollection 2013.

McGill BJ, Enquist BJ, Weiher E, Westoby M (2006). Rebuilding community ecology from functional traits. Trends in Ecology Evolution, 21, 178-185

Nihlgard B (1985). The ammonium hypothesis-An additional explanation to the forest dieback in Europe. AMBIO, 14, 2-8

Oguchi R, Hikosaka K, Hirose T (2003). Does the photosynthetic light-acclimation need change in leaf anatomy? Plant, Cell \& Environment, 26, 505-512.

Ono K, Nishi Y, Watanable A, Terashima I (2001). Possible mechanisms of adaptive leaf senescence. Plant Biology, 3, 234-243.

Ordoñez JC, van Bodegom PM, Witte JPM, Wright IJ, Reich PB, Aerts R (2009). A global study of relationships between leaf traits, climate and soil measures of nutrient fertility. Global Ecology and Biogeography, 18, 137-149.

Osnas JLD, Lichstein JW, Reich PB, Pacala SW (2013). Global leaf trait relationships: Mass, area, and the leaf economics spectrum. Science, 340, 741-744.

Pensa M, Karu H, Luud A, Kund K (2010). Within-species correlations in leaf traits of three boreal plant species along a latitudinal gradient. Plant Ecology, 208, 155-166.

Pontes LDS, Soussana JF, Louault F, Andueza D, Carrère P (2007). Leaf traits affect the above-ground productivity and quality of pasture grasses. Functional Ecology, 21, 844-853.

Reich PB, Oleksyn J (2004). Global patterns of plant leaf N and $\mathrm{P}$ in relation to temperature and latitude. Proceedings of the National Academy of Sciences of the United States of America, 101, 11001-11006.

Reich PB, Wright IJ, Cavender-Bares J, Craine JM, Oleksyn J, Westoby M, Walters MB (2003). The evolution of plant functional variation: Traits, spectra, and strategies. International Journal of Plant Sciences, 164(S3), S143-S164.

Richter A, Burrows JP, Nüss H, Granier C, Niemeier U (2005). Increase in tropospheric nitrogen dioxide over China observed from space. Nature, 437, 129-132.

Rose L, Rubarth MC, Hertel D, Leuschner C (2013). Management alters interspecific leaf trait relationships and trait-based species rankings in permanent meadows. Journal of Vegetation Science, 24, 239-250.

Royer DL (2009). Nutrient turnover rates in ancient terrestrial ecosystems. Palaios, 23, 421-423.

Royer DL, Miller IM, Peppe DJ, Hickey LJ (2010). Leaf economic traits from fossils support a weedy habit for early angiosperms. American Journal of Botany, 97, 438-445.

Scoffoni C, Rawls M, McKown A, Cochard H, Sack L (2011). Decline of leaf hydraulic conductance with dehydration:
Relationship to leaf size and venation architecture. Plant Physiology, 156, 832-843.

Shipley B, Lechowicz MJ, Wright I, Reich PB (2006). Fundamental trade-offs generating the worldwide leaf economics spectrum. Ecology, 87, 535-541.

Silvertown J, Franco FB, Harper JL (1997). Plant Life Histories: Ecology, Phylogeny and Evolution. Cambridge University Press, Cambridge, UK.

Suding KN, Collins SL, Gough L, Clark C, Cleland EE, Gross KL, Milchunas DG, Pennings S (2005). Functional- and abundance-based mechanisms explain diversity loss due to $\mathrm{N}$ fertilization. Proceedings of the National Academy of Sciences of the United States of America, 102, 4387-4392.

Sultan SE (2000). Phenotypic plasticity for plant development, function and life history. Trends in Plant Science, 5, 537-542.

Tamm CO (1990). Nitrogen in Terrestrial Ecosystems: Questions of Productivity, Vegetational Change, and Ecological Stability. Springer, Berlin. 116.

Ti CP, Yan XY (2010). Estimation of atmospheric nitrogen wet deposition in China mainland from based on $\mathrm{N}$ emission data. Journal of Agro-Environment Science, 29, 16061611. (in Chinese with English abstract) [遆超普, 颜晓元 (2010). 基于氮排放数据的中国大陆大气氮素湿沉降量 估算. 农业环境科学学报, 29, 1606-1611.]

Ulrich B (1995). The history and possible causes of forest decline in central Europe, with particular attention to the German situation. Environmental Reviews, 3, 262-276.

Vitousek PM, Aber JD, Howarth RW, Likens GE, Matson PA, Schindler DW, Schlesinger WH, Tilman DG (1997). Human alteration of the global nitrogen cycle: Sources and consequences. Ecological Applications, 7, 737-750.

Wan HW, Yang Y, Bai SQ, Xu YH, Bai YF (2008). Variations in leaf functional traits of six species along a nitrogen addition gradient in Leymus chinensis steppe in Inner Mongolia. Journal of Plant Ecology (Chinese Version), 32, 611-621. (in Chinese with English abstract) [万宏伟, 杨 阳, 白世勤, 徐云虎, 白永飞 (2008). 羊草草原群落6种 植物叶片功能特性对氮素添加的响应. 植物生态学报, 32, 611-621.]

Wang CS, Wang SP (2015). A review of research on responses of leaf traits to climate change. Chinese Journal of Plant Ecology, 39, 206-216. (in Chinese with English abstract) [王常顺, 汪诗平 (2015). 植物叶片性状对气候变化的 响应研究进展. 植物生态学报, 39, 206-216.]

Westoby M, Falster DS, Moles AT, Vesk PA, Wright IJ (2002). Plant ecological strategies: Some leading dimensions of variation between species. Annual Review of Ecology and Systematics, 33, 125-159.

Wilson PJ, Thompson K, Hodgson JG (1999). Specific leaf area and leaf dry matter content as alternative predictors of plant strategies. New Phytologist, 143, 155-162. 
Wright IJ, Reich PB, Cornelissen JHC, Falster DS, Groom PK, Hikosaka K, Lee W, Lusk CH, Niinemet Ülo, Oleksyn J, Osada N, Poorter H, Warton DI, Westoby M (2005). Modulation of leaf economic traits and trait relationships by climate. Global Ecology and Biogeography, 14, 411-421.

Wright IJ, Reich PB, Westoby M (2001). Strategy shifts in leaf physiology, structure and nutrient content between species of high- and low-rainfall and high- and low-nutrient habitats. Functional Ecology, 15, 423-434.

Wright IJ, Reich PB, Westoby M, Ackerly DD, Baruch Z, Bongers F, Cavender-Bares J, Chapin T, Cornelissen JHC, Diemer M, Flexas J, Garnier E, Groom PK, Gulias J, Hikosaka K, Lamont BB, Lee T, Lee W, Lusk C, Midgley JJ, Navas ML, Niinemets U, Oleksyn J, Osada N, Poorter H, Poot P, Prior L, Pyankov VI, Roumet C, Thomas SC, Tjoelker MG, Veneklaas EJ, Villar R (2004). The worldwide leaf economics spectrum. Nature, 428, 821-827.

Wright JP, Sutton-Grier A (2012). Does the leaf economic spectrum hold within local species pools across varying environmental conditions? Functional Ecology, 26, 1390-1398.

Wright RF, Rasmussen L (1998). Introduction to the NITREX and EXMAN projects. Forest Ecology and Management, 101, 1-7.

Wyka T, Robakowski P, Zytkowiak R (2000). Leaf age as a factor in anatomical and physiological acclimative responses of Taxus baccata L. needles to contrasting irradiance environments. Photosynthesis Research, 95, 87-99.

Zhang JL, Zhu JJ, Cao KF (2007). Seasonal variation in photosynthesis in six woody species with different leaf phenology in a valley savanna in southwestern China. Trees, 21, 631-643.

Zhang WN, Liao ZY (2009). Research advances in the effect of nitrogen deposition on forest plants. Environmental Science Survey, 28(3), 21-24. (in Chinese) [张维娜, 廖周 瑜 (2009). 氮沉降增加对森林植物影响的研究进展. 环 境科学导刊, 28(3), 21-24.]

Zhao XF, Xu HL, Zhang P, Zhang QQ (2014). Influence of nutrient and water additions on functional traits of Salsola nitraria in desert grassland. Chinese Journal of Plant Ecology, 38, 134-146. (in Chinese with English abstract) [赵新风 徐海量, 张鹏, 张青青 (2014). 养分与水分添 加对荒漠草地植物钠猪毛菜功能性状的影响. 植物生 态学报, 38, 134-146.]

Zheng XH, Fu CB, Xu XK, Yan XD, Huang Y, Han SH, Hu F, Chen GX (2002). The Asian nitrogen cycle case study. AMBIO, 31(2), 79-87.

责任编委: 石培礼 责任编辑: 王 葳

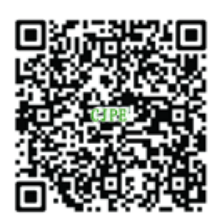

植物生态学报官网

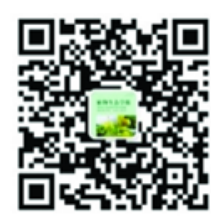

微信订阅号

期刊及学科

相关信息发布

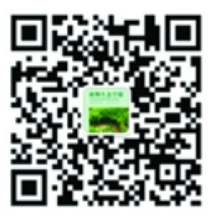

微信服务号

稿件状态查询 全文检索汶览 